\title{
New scheme for color confinement and violation of the non-Abelian Bianchi identities
}

\author{
Tsuneo Suzuki, ${ }^{1, *}$ Katsuya Ishiguro, ${ }^{2}$ and Vitaly Bornyakov ${ }^{3}$ \\ ${ }^{1}$ Kanazawa University, Kanazawa 920-1192, Japan \\ ${ }^{2}$ Library and Information Technology, Kochi University, Kochi 780-8520, Japan \\ ${ }^{3}$ NRC "Kurchatov Institute"-IHEP, 142281 Protvino, Russia and School of Biomedicine, Far Eastern \\ Federal University, Vladivostok 690950, Russia
}

(Received 23 November 2017; published 5 February 2018)

\begin{abstract}
A new scheme for color confinement in QCD due to violation of the non-Abelian Bianchi identities is proposed. The violation of the non-Abelian Bianchi identities (VNABI) $J_{\mu}$ is equal to Abelian-like monopole currents $k_{\mu}$ defined by the violation of the Abelian-like Bianchi identities. Although VNABI is an adjoint operator satisfying the covariant conservation law $D_{\mu} J_{\mu}=0$, it satisfies, at the same time, the Abelian-like conservation law $\partial_{\mu} J_{\mu}=0$. The Abelian-like conservation law $\partial_{\mu} J_{\mu}=0$ is also gaugecovariant. There are $N^{2}-1$ conserved magnetic charges in the case of color $S U(N)$. The charge of each component of VNABI is quantized à la Dirac. The color-invariant eigenvalues $\lambda_{\mu}$ of VNABI also satisfy the Abelian conservation law $\partial_{\mu} \lambda_{\mu}=0$ and the magnetic charges of the eigenvalues are also quantized à la Dirac. If the color invariant eigenvalues condense in the QCD vacuum, each color component of the nonAbelian electric field $E^{a}$ is squeezed by the corresponding color component of the solenoidal current $J_{\mu}^{a}$. Then only the color singlets alone can survive as a physical state and non-Abelian color confinement is realized. This confinement picture is completely new in comparison with the previously studied monopole confinement scenario based on an Abelian projection after some partial gauge-fixing, where Abelian neutral states can survive as physical. To check if the scenario is realized in nature, numerical studies are done in the framework of lattice field theory by adopting pure $S U(2)$ gauge theory for simplicity. Considering $J_{\mu}(x)=k_{\mu}(x)$ in the continuum formulation, we adopt an Abelian-like definition of a monopole following DeGrand-Toussaint as a lattice version of VNABI, since the Dirac quantization condition of the magnetic charge is satisfied on lattice partially. To reduce severe lattice artifacts, we introduce various techniques of smoothing the thermalized vacuum. Smooth gauge fixings such as the maximal center gauge (MCG), block-spin transformations of Abelian-like monopoles and extraction of physically important infrared long monopole loops are adopted. We also employ the tree-level tadpole improved gauge action of $S U(2)$ gluodynamics. With these various improvements, we measure the density of lattice VNABI: $\rho(a(\beta), n)=\sum_{\mu, s_{n}} \sqrt{\sum_{a}\left(k_{\mu}^{a}\left(s_{n}\right)\right)^{2}} /\left(4 \sqrt{3} V_{n} b^{3}\right)$, where $k_{\mu}^{a}\left(s_{n}\right)$ is an $n$ blocked monopole in the color direction $a, n$ is the number of blocking steps, $V_{n}=V / n^{4}(b=n a(\beta))$ is the lattice volume (spacing) of the blocked lattice. Beautiful and convincing scaling behaviors are seen when we plot the density $\rho(a(\beta), n)$ versus $b=n a(\beta)$. A single universal curve $\rho(b)$ is found from $n=1$ to $n=12$, which suggests that $\rho(a(\beta), n)$ is a function of $b=n a(\beta)$ alone. The universal curve seems independent of a gauge fixing procedure used to smooth the lattice vacuum since the scaling is obtained in all gauges adopted. The scaling, if it exists also for $n \rightarrow \infty$, shows that the lattice definition of VNABI has the continuum limit and the new confinement scenario is realized.
\end{abstract}

DOI: 10.1103/PhysRevD.97.034501

\section{INTRODUCTION}

*suzuki04@staff.kanazawa-u.ac.jp

Published by the American Physical Society under the terms of the Creative Commons Attribution 4.0 International license. Further distribution of this work must maintain attribution to the author(s) and the published article's title, journal citation, and DOI. Funded by SCOAP ${ }^{3}$.
Color confinement in quantum chromodynamics (QCD) is still an important unsolved problem [1].

As a picture of color confinement, 't Hooft [2] and Mandelstam [3] conjectured that the QCD vacuum is a kind of a magnetic superconducting state caused by condensation of magnetic monopoles and an effect dual to the Meissner effect works to confine color charges. However, 
in contrast to SUSY QCD [4] or Georgi-Glashow model $[5,6]$ with scalar fields, to find color magnetic monopoles which condense is not straightforward in QCD.

An interesting idea to realize this conjecture is to project QCD to the Abelian maximal torus group by a partial (but singular) gauge fixing [7]. In $S U(3)$ QCD, the maximal torus group is Abelian $U(1)^{2}$. Then color magnetic monopoles appear as a topological object. Condensation of the monopoles causes the dual Meissner effect [8-10].

Numerically, an Abelian projection in nonlocal gauges such as the maximally Abelian (MA) gauge [11-13] has been found to support the Abelian confinement scenario beautifully [14-20]. Also the Abelian dominance and the dual Meissner effect are observed clearly in local unitary gauges such as F12 and Polyakov (PL) gauges [21].

However, although numerically interesting, the idea of Abelian projection [7] is theoretically very unsatisfactory. (1) In nonperturbative QCD, any gauge-fixing is not necessary at all. There are infinite ways of such a partial gauge-fixing and whether the 't Hooft scheme is gauge independent or not is not known. (2) After an Abelian projection, only one [in $S U(2)$ ] or two [in $S U(3)$ ] gluons are photon-like with respect to the residual $U(1)$ or $U(1)^{2}$ symmetry and the other gluons are massive charged matter fields. Such an asymmetry among gluons is unnatural. (3) How to construct Abelian monopole operators in a gauge-independent way in terms of original gluon fields is not clear at all.

In this paper, we propose a new theoretical scheme for color confinement based on the dual Meissner effect which is free from the above problems. The idea was first expressed by one of the authors (T.S.) in Ref. [22].

Then to check if the above scheme is realized in nature, we study the proposal in the framework of the non-Abelian lattice gauge theory. For simplicity we adopt pure $S U(2)$ lattice gauge theory. First considering $J_{\mu}(x)=k_{\mu}(x)$ in the continuum, we define VNABI on lattice as an Abelian-like monopole following DeGrand-Toussaint [23]. Then as a most important point to be clarified, we are going to study if the lattice VNABI has the non-trivial continuum limit, namely if the scaling of the density exists.

The lattice monopoles exist as a closed loop due to the current conservation law. As shown later explicitly, monopole closed loops are contaminated by lattice artifacts. Hence it is absolutely necessary to introduce various techniques avoiding such large lattice artifacts in order to analyze especially such a quantity as the monopole density, since all lattice artifacts contribute positively to the density. We introduce various techniques of smoothing the thermalized vacuum. Smooth gauge fixings such as the maximal center gauge (MCG) [24,25], block-spin transformations of Abelian-like monopoles, and extraction of physically important infrared long monopoles are taken into account. We also employ the tree-level tadpole improved gauge action.

\section{A NEW CONFINEMENT SCHEME BASED ON VNABI}

\section{A. Equivalence of $\boldsymbol{J}_{\boldsymbol{\mu}}$ and $\boldsymbol{k}_{\boldsymbol{\mu}}$}

First of all, we prove that the Jacobi identities of covariant derivatives lead us to conclusion that violation of the non-Abelian Bianchi identities (VNABI) $J_{\mu}$ is nothing but an Abelian-like monopole $k_{\mu}$ defined by violation of the Abelian-like Bianchi identities without gauge-fixing. Define a covariant derivative operator $D_{\mu}=\partial_{\mu}-i g A_{\mu}$. The Jacobi identities are expressed as

$$
\epsilon_{\mu \nu \rho \sigma}\left[D_{\nu},\left[D_{\rho}, D_{\sigma}\right]\right]=0 .
$$

By direct calculations, one gets

$$
\begin{aligned}
{\left[D_{\rho}, D_{\sigma}\right] } & =\left[\partial_{\rho}-i g A_{\rho}, \partial_{\sigma}-i g A_{\sigma}\right] \\
& =-i g\left(\partial_{\rho} A_{\sigma}-\partial_{\sigma} A_{\rho}-i g\left[A_{\rho}, A_{\sigma}\right]\right)+\left[\partial_{\rho}, \partial_{\sigma}\right] \\
& =-i g G_{\rho \sigma}+\left[\partial_{\rho}, \partial_{\sigma}\right]
\end{aligned}
$$

where the second commutator term of the partial derivative operators can not be discarded, since gauge fields may contain a line singularity. Actually, it is the origin of the violation of the non-Abelian Bianchi identities (VNABI) as shown in the following. The non-Abelian Bianchi identities and the Abelian-like Bianchi identities are, respectively: $D_{\nu} G_{\mu \nu}^{*}=0$ and $\partial_{\nu} f_{\mu \nu}^{*}=0$. The relation $\left[D_{\nu}, G_{\rho \sigma}\right]=D_{\nu} G_{\rho \sigma}$ and the Jacobi identities (1) lead us to

$$
\begin{aligned}
D_{\nu} G_{\mu \nu}^{*} & =\frac{1}{2} \epsilon_{\mu \nu \rho \sigma} D_{\nu} G_{\rho \sigma} \\
& =-\frac{i}{2 g} \epsilon_{\mu \nu \rho \sigma}\left[D_{\nu},\left[\partial_{\rho}, \partial_{\sigma}\right]\right] \\
& =\frac{1}{2} \epsilon_{\mu \nu \rho \sigma}\left[\partial_{\rho}, \partial_{\sigma}\right] A_{\nu} \\
& =\partial_{\nu} f_{\mu \nu}^{*},
\end{aligned}
$$

where $f_{\mu \nu}$ is defined as $f_{\mu \nu}=\partial_{\mu} A_{\nu}-\partial_{\nu} A_{\mu}=\left(\partial_{\mu} A_{\nu}^{a}-\right.$ $\left.\partial_{\nu} A_{\mu}^{a}\right) \sigma^{a} / 2$. Namely Eq. (2) shows that the violation of the non-Abelian Bianchi identities is equivalent to that of the Abelian-like Bianchi identities.

Denote the violation of the non-Abelian Bianchi identities as $J_{\mu}$ :

$$
J_{\mu}=\frac{1}{2} J_{\mu}^{a} \sigma^{a}=D_{\nu} G_{\mu \nu}^{*}
$$

Equation (3) is gauge covariant and therefore a nonzero $J_{\mu}$ is a gauge-invariant property. An Abelian-like monopole $k_{\mu}$ without any gauge-fixing is defined as the violation of the Abelian-like Bianchi identities: 


$$
k_{\mu}=\frac{1}{2} k_{\mu}^{a} \sigma^{a}=\partial_{\nu} f_{\mu \nu}^{*}=\frac{1}{2} \epsilon_{\mu \nu \rho \sigma} \partial_{\nu} f_{\rho \sigma} .
$$

Equation (2) shows that

$$
J_{\mu}=k_{\mu} .
$$

Several comments are in order.

(1) Equation (5) can be considered as a special case of the important relation derived by Bonati et al. [26] in the framework of an Abelian projection to a simple case without any Abelian projection. Actually it is possible to prove directly without the help of the Jacobi identities

$$
\begin{aligned}
J_{\mu}^{a}-k_{\mu}^{a}= & \operatorname{Tr} \sigma^{a} D_{\nu} G_{\mu \nu}^{*}-\partial_{\nu} f_{\mu \nu}^{* a} \\
= & -i g \operatorname{Tr} \sigma^{a}\left[A_{\nu}, G_{\mu \nu}^{*}\right] \\
& -i g \epsilon_{\mu \nu \rho \sigma} \operatorname{Tr} \sigma^{a}\left[\partial_{\nu} A_{\rho}, A_{\sigma}\right] \\
= & 0 .
\end{aligned}
$$

(2) VNABI $J_{\mu}$ transforms as an adjoint operator, so that does the Abelian-like monopole current $k_{\mu}$. This can be proved also directly. Consider a regular gauge transformation

$$
A_{\mu}^{\prime}=V A_{\mu} V^{\dagger}-\frac{i}{g} \partial_{\mu} V V^{\dagger}
$$

Then

$$
\begin{aligned}
k_{\mu}^{\prime} & =\epsilon_{\mu \nu \rho \sigma} \partial_{\nu} \partial_{\rho} A_{\sigma}^{\prime} \\
& =\epsilon_{\mu \nu \rho \sigma} \partial_{\nu} \partial_{\rho}\left(V A_{\mu} V^{\dagger}-\frac{i}{g} \partial_{\mu} V V^{\dagger}\right) \\
& =V\left(\epsilon_{\mu \nu \rho \sigma} \partial_{\nu} \partial_{\rho} A_{\sigma}\right) V^{\dagger} \\
& =V k_{\mu} V^{\dagger}
\end{aligned}
$$

(3) The above equivalence shows VNABI is essentially Abelian-like. It was already argued that singularities of gauge fields corresponding to VNABI must be Abelian [27], although the reasoning is different.

(4) The covariant conservation law $D_{\mu} J_{\mu}=0$ is proved as follows [26]:

$$
\begin{aligned}
D_{\mu} J_{\mu} & =D_{\mu} D_{\nu} G_{\nu \mu}^{*}=\frac{i g}{2}\left[G_{\nu \mu}, G_{\nu \mu}^{*}\right] \\
& =\frac{i g}{4} \epsilon_{\nu \mu \rho \sigma}\left[G_{\nu \mu}, G_{\rho \sigma}\right]=0
\end{aligned}
$$

where

$$
\partial_{\mu} \partial_{\nu} G_{\mu \nu}^{*}=0
$$

is used. The Abelian-like monopole satisfies the Abelian-like conservation law

$$
\partial_{\mu} k_{\mu}=\partial_{\mu} \partial_{\nu} f_{\mu \nu}^{*}=0
$$

due to the antisymmetric property of the Abelianlike field strength [28]. Hence VNABI satisfies also the same Abelian-like conservation law

$$
\partial_{\mu} J_{\mu}=0 .
$$

Both Eqs. (7) and (10) are compatible, since the difference between both quantities

$$
\begin{aligned}
{\left[A_{\mu}, J_{\mu}\right] } & =\frac{1}{2} \epsilon_{\mu \nu \rho \sigma}\left[A_{\mu}, \partial_{\nu} f_{\rho \sigma}\right] \\
& =\epsilon_{\mu \nu \rho \sigma}\left[A_{\mu}, \partial_{\nu} \partial_{\rho} A_{\sigma}\right] \\
& =-\frac{1}{2} \epsilon_{\mu \nu \rho \sigma} \partial_{\nu} \partial_{\mu}\left[A_{\rho}, A_{\sigma}\right] \\
& =\frac{i}{g}\left(\partial_{\mu} \partial_{\nu} G_{\mu \nu}^{*}-\partial_{\mu} \partial_{\nu} f_{\mu \nu}^{*}\right) \\
& =0
\end{aligned}
$$

where (8) and (9) are used. Hence the Abelian-like conservation relation (10) is also gauge-covariant.

(5) The Abelian-like conservation relation (10) gives us three conserved magnetic charges in the case of color $S U(2)$ and $N^{2}-1$ charges in the case of color $S U(N)$. But these are kinematical relations coming from the derivative with respect to the divergence of an antisymmetric tensor [28]. The number of conserved charges is different from that of the Abelian projection scenario [7], where only $N-1$ conserved charges exist in the case of color $S U(N)$.

\section{B. Dirac quantization condition}

Next we show that the magnetic charges derived from $k_{4}=J_{4}$ satisfy the Dirac quantization condition with respect to magnetic and electric charges. Consider a space-time point $O$ where the Bianchi identities are violated and a three-dimensional sphere $V$ of a large radius $r$ from $O$. Since $k_{4}=J_{4}$ is given by the total derivative, the behavior of the gauge field at the surface of the sphere is relevant. When $r \rightarrow \infty$, the non-Abelian field strength should vanish since otherwise the action diverges. Then the magnetic charge could be evaluated by a gauge field described by a pure gauge $A_{\mu}=\Omega \partial_{\mu} \Omega^{\dagger} /$ ig , where $\Omega$ is a gauge transformation matrix satisfying $\Omega\left[\partial_{\mu}, \partial_{\nu}\right] \Omega^{\dagger}=0$ at $r \rightarrow \infty$. Then the magnetic charge $g_{m}^{d}$ in a color direction is evaluated as follows: 


$$
\begin{aligned}
g_{m}^{d} & =\int_{V} d^{3} x k_{4}^{d}=\int d^{3} x \frac{1}{2} \epsilon_{4 \nu \rho \sigma} \partial_{\nu}\left(\partial_{\rho} A_{\sigma}^{d}-\partial_{\sigma} A_{\rho}^{d}\right) \\
& =\int_{V} d^{3} x \frac{1}{2 i g} \epsilon_{i j k} \partial_{i} \operatorname{Tr} \sigma^{d}\left(\partial_{j} \Omega \partial_{k} \Omega^{\dagger}-\partial_{k} \Omega \partial_{j} \Omega^{\dagger}+\Omega\left[\partial_{j}, \partial_{k}\right] \Omega^{\dagger}\right) \\
& =\int_{V} d^{3} x \frac{1}{2 g} \epsilon_{i j k}\left\{\epsilon^{a b c} \partial_{i}\left(\hat{\phi}^{a} \partial_{j} \hat{\phi}^{b} \partial_{k} \hat{\phi}^{c}+\partial_{i} \operatorname{Tr} \sigma^{d} \Omega\left[\partial_{j}, \partial_{k}\right] \Omega^{\dagger}\right)\right\} \\
& =\int_{\partial V} d^{2} S \frac{1}{2 g} \epsilon_{i j k} \epsilon^{a b c} \hat{\phi}^{a} \partial_{j} \hat{\phi}^{b} \partial_{k} \hat{\phi}^{c},
\end{aligned}
$$

where $\Omega\left[\partial_{j}, \partial_{k}\right] \Omega^{\dagger}=0$ on the surface at $r \rightarrow \infty$ is used and $\hat{\phi}$ is a Higgs-like field defined as

$$
\begin{aligned}
\hat{\phi} & =\hat{\phi}^{i} \sigma^{i} \\
& =\Omega \sigma^{d} \Omega^{\dagger}
\end{aligned}
$$

$\hat{\phi}^{2}=1$ is shown easily. Since the field $\hat{\phi}$ is a single-valued function, Eq. (11) is given by the wrapping number $n$ characterizing the homotopy class of the mapping between the spheres described by $\hat{\phi}^{2}=\left(\hat{\phi}^{1}\right)^{2}+\left(\hat{\phi}^{2}\right)^{2}+\left(\hat{\phi}^{3}\right)^{2}=1$ and $\partial V=S^{2}: \pi_{2}\left(S^{2}\right)=Z$. Namely

$$
g_{m}^{d} g=4 \pi n .
$$

This is just the Dirac quantization condition. Note that the minimal color electric charge in any color direction is $g / 2$. Hence the kinematical conservation law is also topological.
What happens in the case of color $S U(3)$ ? Then it is easy to prove that the three $S U(2)$ subspaces (isospin, U-spin, V-spin) play the role in the above mapping and all eight magnetic charges are quantized similarly à la Dirac. In $S U(3)$, a gauge field for $r \rightarrow \infty$ becomes $A_{\mu}=\Omega \partial_{\mu} \Omega^{\dagger} / i g$, where now $\Omega$ is a $3 \times 3$ gauge transformation matrix of $S U(3)$. Then for example, consider a magnetic charge in the $\lambda^{1}$ direction, where $\lambda^{a}$ is the Gell-Mann matrix. Then define a Higgs-like field $\hat{\phi}$ as

$$
\begin{aligned}
\hat{\phi} & =\hat{\phi}^{i} \lambda^{i} \\
& =\Omega \lambda^{1} \Omega^{\dagger} .
\end{aligned}
$$

The magnetic charge $g_{m}^{1}$ in the $\lambda^{1}$ color direction is evaluated as follows:

$$
\begin{aligned}
g_{m}^{1} & =\int_{V} d^{3} x k_{4}^{1}=\int d^{3} x \frac{1}{2} \epsilon_{4 \nu \rho \sigma} \partial_{\nu}\left(\partial_{\rho} A_{\sigma}^{1}-\partial_{\sigma} A_{\rho}^{1}\right) \\
& =\int_{V} d^{3} x \frac{1}{2 i g} \epsilon_{i j k} \partial_{i} \operatorname{Tr} \lambda^{1}\left(\partial_{j} \Omega \partial_{k} \Omega^{\dagger}-\partial_{k} \Omega \partial_{j} \Omega^{\dagger}+\Omega\left[\partial_{j}, \partial_{k}\right] \Omega^{\dagger}\right) \\
& =\int_{V} d^{3} x \frac{1}{2 g} \epsilon_{i j k}\left\{\epsilon^{a b c} \partial_{i}\left(\hat{\phi}^{a} \partial_{j} \hat{\phi}^{b} \partial_{k} \hat{\phi}^{c}+\partial_{i} \operatorname{Tr} \lambda^{1} \Omega\left[\partial_{j}, \partial_{k}\right] \Omega^{\dagger}\right)\right\} \\
& =\int_{\partial V} d^{2} S \frac{1}{2 g} \epsilon_{i j k} \epsilon^{a b c} \hat{\phi}^{a} \partial_{j} \hat{\phi}^{b} \partial_{k} \hat{\phi}^{c} .
\end{aligned}
$$

Now one sees from (14)

$$
\hat{\phi}^{2}=\left(\begin{array}{lll}
1 & 0 & 0 \\
0 & 1 & 0 \\
0 & 0 & 0
\end{array}\right) .
$$

The condition (16) gives us

$$
\begin{aligned}
& \left(\hat{\phi}^{1}\right)^{2}+\left(\hat{\phi}^{2}\right)^{2}+\left(\hat{\phi}^{3}\right)^{2}=1, \\
& \hat{\phi}^{4}=\hat{\phi}^{5}=\hat{\phi}^{6}=\hat{\phi}^{7}=\hat{\phi}^{8}=0 .
\end{aligned}
$$

Namely the subspace composed of $\left(\hat{\phi}^{1}, \hat{\phi}^{2}, \hat{\phi}^{3}\right)$ is a sphere $S^{2}$ and the mapping is just like that in the case of color $S U(2)$. Hence $g_{m}^{1}$ satisfies the Dirac quantization condition

$$
g_{m}^{1} g=4 \pi n .
$$

The same condition holds good for all other magnetic charges.

\section{Proposal of the vacuum in the confinement phase}

Now we propose a new mechanism of color confinement in which VNABI $J_{\mu}$ play an important role in the vacuum. 
Since VNABI transforms as an adjoint operator, it can be diagonalized by a unitary matrix $V_{d}(x)$ as follows:

$$
V_{d}(x) J_{\mu}(x) V_{d}^{\dagger}(x)=\lambda_{\mu}(x) \frac{\sigma_{3}}{2},
$$

where $\lambda_{\mu}(x)$ is the eigenvalue of $J_{\mu}(x)$ and is then color invariant but magnetically charged. Note that $V_{d}(x)$ does not depend on $\mu$ due to the Coleman-Mandula theorem $[29,30]$. Then one gets

$$
\begin{gathered}
\Phi(x) \equiv V_{d}^{\dagger}(x) \sigma_{3} V_{d}(x) \\
J_{\mu}(x)=\frac{1}{2} \lambda_{\mu}(x) \Phi(x), \\
\sum_{a}\left(J_{\mu}^{a}(x)\right)^{2}=\sum_{a}\left(k_{\mu}^{a}(x)\right)^{2}=\left(\lambda_{\mu}(x)\right)^{2} .
\end{gathered}
$$

Namely the color electrically charged part and the magnetically charged part are separated out. From (19) and (10), one gets

$$
\partial_{\mu} J_{\mu}(x)=\frac{1}{2}\left(\partial_{\mu} \lambda_{\mu}(x) \Phi(x)+\lambda_{\mu}(x) \partial_{\mu} \Phi(x)\right)=0 .
$$

Since $\Phi(x)^{2}=1$,

$$
\partial_{\mu} \lambda_{\mu}(x)=-\lambda_{\mu}(x) \Phi(x) \partial_{\mu} \Phi(x)=0 .
$$

Hence the eigenvalue $\lambda_{\mu}$ itself satisfies the Abelian conservation rule.

Furthermore, when use is made of (6), it is possible to prove that

$$
\frac{1}{2} \epsilon_{\mu \nu \rho \sigma} \partial_{\nu} f_{\mu \nu}^{\prime}(x)=\lambda_{\mu}(x) \frac{\sigma_{3}}{2}
$$

where

$$
\begin{aligned}
f_{\mu \nu}^{\prime}(x) & =\partial_{\mu} A_{\nu}^{\prime}(x)-\partial_{\nu} A_{\mu}^{\prime}(x) \\
A_{\mu}^{\prime} & =V_{d} A_{\mu} V_{d}^{\dagger}-\frac{i}{g} \partial_{\mu} V_{d} V_{d}^{\dagger}, \\
& \equiv \frac{A_{\mu}^{\prime a} \sigma^{a}}{2} .
\end{aligned}
$$

Namely,

$$
\begin{gathered}
\frac{1}{2} \epsilon_{\mu \nu \rho \sigma} \partial_{\nu} f_{\rho \sigma}^{\prime 1,2}(x)(x)=0 \\
\frac{1}{2} \epsilon_{\mu \nu \rho \sigma} \partial_{\nu} f_{\rho \sigma}^{\prime 3}(x)(x)=\lambda_{\mu}(x) .
\end{gathered}
$$

The singularity appears only in the diagonal component of the gauge field $A_{\mu}^{\prime}$.
If one considers for large $r$

$$
\begin{aligned}
& A_{\mu}^{\prime} \rightarrow \Omega \partial_{\mu} \Omega^{\dagger} / i g, \\
& \hat{\phi}=\hat{\phi}^{i} \sigma^{i}=\Omega \sigma^{3} \Omega^{\dagger},
\end{aligned}
$$

one can easily see from (24) and (11) that the magnetic charge from the eigenvalue $\lambda_{\mu}$ also satisfies the Dirac quantization condition (12).

It is very interesting to see that $f_{\mu \nu}^{\prime 3}(x)$ is actually the gauge invariant 'tHooft tensor [5]:

$$
f_{\mu \nu}^{\prime 3}(x)=\operatorname{Tr} \Phi(x) G_{\mu \nu}(x)+\frac{i}{2 g} \operatorname{Tr} \Phi(x) D_{\mu} \Phi(x) D_{\nu} \Phi(x),
$$

in which the field $\Phi(x)$ (18) plays a role of the scalar Higgs field in Ref. [5]. To be noted is that the field $\Phi(x)$ (18) is determined uniquely by VNABI itself in the gluodynamics without any Higgs field. In this sense, our scheme can be regarded as a special Abelian projection scenario with the partial gauge-fixing condition where $J_{\mu}(x)$ are diagonalized. The condensation of the gauge-invariant magnetic currents $\lambda_{\mu}$ does not give rise to a spontaneous breaking of the color electric symmetry. Condensation of the color invariant magnetic currents $\lambda_{\mu}$ may be a key mechanism of the physical confining vacuum.

The main difference between our new scheme and previous Abelian projection schemes is that in the former there exist $N^{2}-1$ conserved magnetic currents squeezing $N^{2}-1$ color electric fields and color (not charge) confinement is shown explicitly, whereas in the latter, there exists only $N-1$ conserved currents giving charge confinement. In our scheme, the $N^{2}-1$ conserved magnatic currents are degenerate in the vacuum to $N-1$ color-invariant currents corresponding to the eigenvalues. To show the difference of this scheme from the previous 'tHooft Abelian projection with some partial gauge-fixing, we show Table I in which typical different points are written.

Let us make a comment here on the relation derived by Bonati et al.[26]:

$$
k_{\mu}^{A B}(x)=\operatorname{Tr}\left\{J_{\mu}(x) \Phi^{A B}(x)\right\},
$$

where $k_{\mu}^{A B}(x)$ is an Abelian monopole, $\Phi^{A B}(x)=$ $V_{A B}^{\dagger}(x) \sigma_{3} V_{A B}(x)$ and $V_{A B}(x)$ is a partial gauge-fixing matrix in some Abelian projection like the MA gauge. Making use of Eq. (19), we get

$$
k_{\mu}^{A B}(x)=\lambda_{\mu}(x) \tilde{\Phi}^{3}(x)
$$

where

$$
\tilde{\Phi}(x)=V_{A B}(x) V_{d}^{\dagger}(x) \sigma_{3} V_{A B}^{\dagger}(x) V_{d}(x)=\tilde{\Phi}^{a}(x) \sigma^{a} .
$$

The relation (25) is important, since existence of an Abelian monopole in any Abelian projection scheme is guaranteed 
TABLE I. Comparison between the 'tHooft Abelian projection studies and the present work in $S U(2)$ QCD. $\hat{\phi}^{\prime}=V_{p}^{\dagger} \sigma_{3} V_{p}$, where $V_{p}$ is a partial gauge-fixing matrix of an Abelian projection. $\left(u_{c}, d_{c}\right)$ is a color-doublet quark pair. MA means maximally Abelian.

\begin{tabular}{|c|c|c|}
\hline & $\begin{array}{l}\text { The 'tHooft Abelian projection scheme } \\
\text { Previous works [11-21] } \\
\text { Reference [26] }\end{array}$ & This work and Refs. [32,33] \\
\hline Origin of $k_{\mu}$ & A singular gauge transformation & $k_{\mu}^{a}=J_{\mu}^{a}$ \\
\hline No. of conserved $k_{\mu}$ & 1 & 3 \\
\hline Role of $A_{\mu}^{a}$ & One photon $A_{\mu}^{3}$ with $k_{\mu}^{3}+2$ massive $A_{\mu}^{ \pm}$ & Three gluons $A_{\mu}^{a}$ with $k_{\mu}^{a}$ \\
\hline Flux squeezing & One electric field $E_{\mu}$ & Three electric fields $E_{-}^{a}$ \\
\hline Number of physical mesons & 2 Abelian neutrals, $\bar{u}_{c} u_{c}$ and $\bar{d}_{c} d_{c}$ & 1 color singlet $\bar{u}_{c} u_{c}+\bar{d}_{c} d_{c}$ \\
\hline Expected confining vacuum & Condensation of Abelian monopoles & Condensation of color-invariant $\lambda_{\mu}$ \\
\hline Privileged gauge choice & A singular gauge & No need of gauge-fixing \\
\hline
\end{tabular}

by that of VNABI $J_{\mu}$ in the continuum limit. Hence if in any special gauge such as MA gauge, Abelian monopoles remain non-vanishing in the continuum as suggested by many numerical data [14-20], VNABI also remain nonvanishing in the continuum.

\section{LATTICE NUMERICAL STUDY OF THE CONTINUUM LIMIT}

\section{A. Definition of VNABI on lattice}

Let us try to define VNABI on lattice. In the previous section, VNABI $J_{\mu}(x)$ is shown to be equivalent in the continuum limit to the violation of the Abelian-like Bianchi identities $J_{\mu}(x)=k_{\mu}(x)$.

On lattice, we have to define a quantity which leads us to the above VNABI in the continuum limit. There are two possible definitions which lead us to the above VNABI in the naive continuum limit. One is a quantity keeping the adjoint transformation property under the lattice $S U(2)$ gauge transformation $V(s)$ :

$$
U(s, \mu)^{\prime}=V(s) U(s, \mu) V^{\dagger}(s+\mu) .
$$

Here $U(s, \mu)$ is a lattice gauge link field. Such a quantity was proposed in Ref. [34]:

$$
\begin{aligned}
J_{\mu}(s) & \equiv \frac{1}{2}\left(U(s, \nu) U_{\mu \nu}(s+\nu) U^{\dagger}(s, \nu)-U_{\mu \nu}(s)\right), \\
U_{\mu \nu}(s) & \equiv U(s, \mu) U(s+\mu, \nu) U^{\dagger}(s+\nu, \mu) U^{\dagger}(s, \nu)
\end{aligned}
$$

where $U_{\mu \nu}(s)$ is a plaquette variable corresponding to the non-Abelian field strength. This transforms as an adjoint operator:

$$
J_{\mu}^{\prime}(s)=V(s) J_{\mu}(s) V^{\dagger}(s)
$$

and satisfies the covariant conservation law

$$
\begin{aligned}
\sum_{\mu} D_{\mu}^{L} J_{\mu}(s) & =\sum_{\mu}\left(U(s+\mu, \mu) J_{\mu}(s) U^{\dagger}(s, \mu)-J_{\mu}(s)\right) \\
& =0 .
\end{aligned}
$$

However it does not satisfy the Abelian conservation law:

$$
\sum_{\mu}\left(J_{\mu}(s)-J_{\mu}(s-\mu)\right)=0 .
$$

Moreover it does not have a property corresponding to the Dirac quantization condition satisfied by the continuum VNABI. The last point is very unsatisfactory, since the topological property as a monopole is essential.

Hence we adopt here the second possibility which can reflect partially the topological property satisfied by VNABI. That is, we define VNABI on lattice as the Abelian-like monopole [32,33] following DeGrand and Toussaint [23]. First we define Abelian link and plaquette variables:

$$
\begin{gathered}
\theta_{\mu}^{a}(s)=\arctan \left(U_{\mu}^{a}(s) / U_{\mu}^{0}(s)\right) \quad\left(\left|\theta_{\mu}^{a}(s)\right|<\pi\right) \\
\theta_{\mu \nu}^{a}(s) \equiv \partial_{\mu} \theta_{\nu}^{a}(s)-\partial_{\nu} \theta_{\mu}^{a}(s),
\end{gathered}
$$

where $\partial_{\nu}\left(\partial_{\nu}^{\prime}\right)$ is a forward (backward) difference. Then the plaquette variable can be decomposed as follows:

$$
\theta_{\mu \nu}^{a}(s)=\bar{\theta}_{\mu \nu}^{a}(s)+2 \pi n_{\mu \nu}^{a}(s) \quad\left(\left|\bar{\theta}_{\mu \nu}^{a}\right|<\pi\right),
$$

where $n_{\mu \nu}^{a}(s)$ is an integer corresponding to the number of the Dirac string. Then VNABI as Abelian monopoles is defined by

$$
\begin{aligned}
k_{\mu}^{a}(s) & =-(1 / 2) \epsilon_{\mu \alpha \beta \gamma} \partial_{\alpha} \bar{\theta}_{\beta \gamma}^{a}(s+\hat{\mu}) \\
& =(1 / 2) \epsilon_{\mu \alpha \beta \gamma} \partial_{\alpha} n_{\beta \gamma}^{a}(s+\hat{\mu}) \\
J_{\mu}(s) & \equiv \frac{1}{2} k_{\mu}^{a}(s) \sigma^{a} .
\end{aligned}
$$

This definition (32) of VNABI satisfies the Abelian conservation condition (28) and takes an integer value which corresponds to the magnetic charge obeying the Dirac quantization condition. The eigenvalue $\lambda_{\mu}$ is defined from (20) as 


$$
\left(\lambda_{\mu}(s)\right)^{2}=\sum_{a}\left(k_{\mu}^{a}(s)\right)^{2}
$$

However it does not satisfy the transformation property (27) on the lattice. We will demonstrate that this property is recovered in the continuum limit by showing the gauge invariance of the monopole density or the squared monopole density (33) in the scaling limit.

\section{B. Simulation details}

\section{Tadpole improved gauge action}

First of all, we adopt the tree level improved action of the form [35] for simplicity in $S U(2)$ gluodynamics:

$$
S=\beta \sum_{p l} S_{p l}-\frac{\beta}{20 u_{0}^{2}} \sum_{r t} S_{r t}
$$

where $S_{p l}$ and $S_{r t}$ denote plaquette and $1 \times 2$ rectangular loop terms in the action,

$$
S_{p l, r t}=\frac{1}{2} \operatorname{Tr}\left(1-U_{p l, r t}\right),
$$

the parameter $u_{0}$ is the input tadpole improvement factor taken here equal to the fourth root of the average plaquette $P=\left\langle\frac{1}{2} \operatorname{tr} U_{p l}\right\rangle$. In our simulations we have not included one-loop corrections to the coefficients, for the sake of simplicity.

The lattices adopted are $48^{4}$ for $\beta=3.0-3.9$ and $24^{4}$ for $\beta=3.0-3.7$. The latter was taken mainly for studying finite-size effects. The simulations with the action (34) have been performed with parameters given in Table $\mathrm{V}$ in Appendix A following similarly the method as adopted in Ref. [36].

\section{The non-Abelian string tension}

In order to fix the physical lattice scale we need to compute one physical dimensionful observable the value of which is known. For this purpose we choose the string tension $\sigma$. The string tension for the action (34) was computed long ago in [36,37] but we improve this measurement according to present standards. We use the hypercubic blocking (HYP) invented by the authors of Refs. [38-41] to reduce the statistical errors. After one step of HYP, APE smearing [42] were applied to the spacelike links. The spatial smearing is made, as usually, in order to variationally improve the overlap with a mesonic flux tube state. The results of the measured string tensions are listed also in Table V in Appendix A.

\section{Introduction of smooth gauge-fixings}

Monopole loops in the thermalized vacuum produced in the above improved action (34) still contain large amount of lattice artifacts. Hence we here adopt a gauge-fixing technique smoothing the vacuum, although any gaugefixing is not necessary in principle in the continuum limit [43]:

(1) Maximal center gauge (MCG).

The first gauge is the maximal center gauge $[24,25]$ which is usually discussed in the framework of the center vortex idea. We adopt the so-called direct maximal center gauge which requires maximization of the quantity

$$
R=\sum_{s, \mu}(\operatorname{Tr} U(s, \mu))^{2}
$$

with respect to local gauge transformations. The condition (36) fixes the gauge up to $Z(2)$ gauge transformation and can be considered as the Landau gauge for the adjoint representation. In our simulations, we choose simulated annealing algorithm as the gauge-fixing method which is known to be powerful for finding the global maximum. For details, see Ref. [44].

(2) Direct Laplacian center gauge (DLCG).

The second is the Laplacian center gauge [45] which is also discussed in connection to center vortex idea. Here we adopt the so-called direct Laplacian center gauge. First, we require maximization of the quantity

$$
R_{M}=\sum_{s, \mu} \operatorname{Tr}\left[M^{T}(s) U^{A}(s, \mu) M(s, \mu)\right]
$$

where $U^{A}(s, \mu)$ denotes the adjoint representation of $U(s, \mu)$ and $M(s, \mu)$ is a real-valued $3 \times 3$ matrix in $S U(2)$ gauge theory which satisfies the constraint

$$
\frac{1}{V} \sum_{s} \sum_{j} M_{i j}^{T}(s) M_{j k}(s)=\delta_{i k}
$$

with $V$ lattice volume. Matrix field $M(s)$ which leads to a global maximum of $R_{M}$ is composed of the three lowest eigenfunctions of a lattice Laplacian operator. Second, to determine the corresponding gauge transformation, we construct $S O(3)$ matrix-valued field which is the closest to $M(s)$ and satisfies the corresponding Laplacian condition by local gauge transformation. Finally, the $S O(3)$ matrix-valued field is mapped to an $\mathrm{SU}(2)$ matrix-valued field which is used to the gauge transformation for the original lattice gauge field in fundamental representation. After that, DLCG maximizes the quantity (36) with respect to solving a lattice Laplacian equation.

(3) Maximal Abelian Wilson loop gauge (AWL).

Another example of a smooth gauge is introduced. It is the maximal Abelian Wilson loop gauge (AWL) in which 


$$
R=\sum_{s, \mu \neq \nu} \sum_{a}\left(\cos \left(\theta_{\mu \nu}^{a}(s)\right)\right.
$$

is maximized. Here $\theta_{\mu \nu}^{a}(s)$ have been introduced in Eq. (31). Since $\cos \left(\theta_{\mu \nu}^{a}(s)\right)$ are $1 \times 1$ Abelian Wilson loops, the gauge is called as the maximal Abelian Wilson loop gauge (AWL). A similar gauge was proposed in [46], although only one-color component was considered then in comparison with the maximal Abelian gauge (MAG). Note that even $1 \times 1$ small Abelian Wilson loop is enhanced when a smooth gauge condition such as the MA gauge is adopted. The details are presented in the Appendix B.

(iv) Maximal Abelian and $U(1)$ Landau gauge (MAU1).

The fourth is the combination of the maximal Abelian gauge (MAG) and the $U(1)$ Landau gauge $[12,13]$. Namely we first perform the maximal Abelian gauge fixing and then with respect to the remaining $U(1)$ symmetry the Landau gauge fixing is done. This case breaks the global $S U(2)$ color symmetry contrary to the previous three cases (MCG, DLCG, and AWL) but nevertheless we consider this case since the vacuum is smoothed fairly well. MAG is the gauge which maximizes

$$
R=\sum_{s, \hat{\mu}} \operatorname{Tr}\left(\sigma_{3} U(s, \mu) \sigma_{3} U^{\dagger}(s, \mu)\right)
$$

with respect to local gauge transformations. Then there remains $U(1)$ symmetry to which the Landau gauge fixing is applied, i.e., $\sum_{s, \mu} \cos \theta_{\mu}^{3}(s)$ is maximized [47].

\section{Extraction of infrared monopole loops}

An additional improvement is obtained when we extract important long monopole clusters only from total monopole loop distribution. Let us see a typical example of monopole loop distributions in each gauge in comparison with that without any gauge fixing starting from a thermalized vacuum at $\beta=3.6$ on $24^{4}$ lattice. They are shown in Table II. One can find almost all monopole loops are connected and total loop lengths are very large when no gauge fixing (NGF) is applied as shown in the NGF case. On the other hand, monopole loop lengths become much shorter in all smooth gauges discussed here. Also it is found that only one or few loops are long enough and others are very short as observed similarly in old papers in MAG. The long monopole clusters are called as infrared monopoles and they are the key ingredient giving confinement as shown in the old papers [48]. It is important that in addition to MAU1, all other three MCG, DLCG and AWL cases also have similar behaviors. Since small separate monopole loops can be regarded as lattice artifacts, we extract only infrared monopoles alone. Although there observed only
TABLE II. A typical example of monopole loop distributions [Loop length (L) vs Loop number (No.)] for various gauges in one thermalized vacuum on $24^{4}$ lattice at $\beta=3.6$ in the tadpole improved action. Here $I$ and $L$ denote the color component and

\begin{tabular}{|c|c|c|c|c|c|}
\hline $\begin{array}{l}\text { NGF I }=1 \\
\mathrm{~L}\end{array}$ & No & $\begin{array}{c}\mathrm{MCG} I=1 \\
\mathrm{~L}\end{array}$ & No & $\begin{array}{c}\text { DLCG I }=1 \\
\text { L }\end{array}$ & No \\
\hline 4 & 154 & 4 & 166 & 4 & 164 \\
\hline 6 & 20 & 6 & 64 & 6 & 66 \\
\hline 8 & 7 & 8 & 30 & 8 & 28 \\
\hline 10 & 2 & 10 & 13 & 10 & 15 \\
\hline 14 & 1 & 12 & 11 & 12 & 10 \\
\hline 16 & 1 & 14 & 4 & 14 & 3 \\
\hline \multirow[t]{15}{*}{407824} & 1 & 16 & 5 & 16 & 6 \\
\hline & & 18 & 1 & 18 & 2 \\
\hline & & 22 & 2 & 20 & 1 \\
\hline & & 24 & 2 & 22 & 1 \\
\hline & & 28 & 1 & 24 & 2 \\
\hline & & 30 & 1 & 26 & 3 \\
\hline & & 32 & 1 & 30 & 1 \\
\hline & & 34 & 2 & 36 & 1 \\
\hline & & 36 & 1 & 44 & 1 \\
\hline & & 44 & 1 & 48 & 1 \\
\hline & & 46 & 1 & 54 & 1 \\
\hline & & 48 & 1 & 58 & 1 \\
\hline & & 58 & 1 & 124 & 1 \\
\hline & & 124 & 1 & 1106 & 1 \\
\hline & & 2254 & 1 & 1448 & 1 \\
\hline AWL $\mathrm{I}=1$ & & MAU1 $\mathrm{I}=1$ & & MAU1 $\mathrm{I}=3$ & \\
\hline L & No & $\mathrm{L}$ & No & $\mathrm{L}$ & No \\
\hline 4 & 142 & 4 & 73 & 4 & 190 \\
\hline 6 & 66 & 6 & 32 & 6 & 80 \\
\hline 8 & 36 & 8 & 13 & 8 & 22 \\
\hline 10 & 8 & 10 & 11 & 10 & 15 \\
\hline 12 & 7 & 12 & 6 & 12 & 2 \\
\hline 14 & 3 & 14 & 3 & 14 & 3 \\
\hline 16 & 3 & 16 & 2 & 16 & 1 \\
\hline 18 & 1 & 18 & 3 & 18 & 3 \\
\hline 20 & 1 & 20 & 2 & 20 & 3 \\
\hline 22 & 3 & 22 & 1 & 24 & 1 \\
\hline 26 & 3 & 30 & 2 & 36 & 1 \\
\hline 28 & 1 & 34 & 2 & 42 & 1 \\
\hline 30 & 2 & 58 & 1 & 60 & 1 \\
\hline 32 & 1 & 148 & 1 & 66 & 1 \\
\hline 34 & 1 & 5188 & 1 & 146 & 1 \\
\hline 40 & 1 & & & 318 & 1 \\
\hline 46 & 1 & & & 722 & 1 \\
\hline 58 & 1 & & & & \\
\hline 120 & 1 & & & & \\
\hline 308 & 1 & & & & \\
\hline 1866 & 1 & & & & \\
\hline
\end{tabular}
the loop length of the monopole loop, respectively.

one infrared monopole loop in almost all cases, there are some vacua (especially for large beta) having two or three separate long loops which can be seen as infrared one, since they have much longer length than other shorter ones. We here define as infrared monopoles as all loops having loop 
lengths longer than $10 \%$ of the longest one. The cutoff value is not so critical. Actually the definition of infrared loops itself has an ambiguity, since even in the longest loop, we can not separate out some short artifact loops attached accidentally to the real infrared long loop. But such an ambiguity gives us numerically only small effects as seen from the studies of different cutoff values.

\section{Block-spin transformation}

Block-spin transformation and the renormalizationgroup method is known as the powerful tool to study the continuum limit. We introduce the block-spin transformation with respect to Abelian-like monopoles. The idea was first introduced by Ivanenko et al. [49] and applied in obtaining an infrared effective monopole action in Ref. [50]. The $n$ blocked monopole has a total magnetic charge inside the $n^{3}$ cube and is defined on a blocked reduced lattice with the spacing $b=n a, a$ being the spacing of the original lattice. The respective magnetic currents are defined as

$$
\begin{aligned}
k_{\mu}^{(n)}\left(s_{n}\right)= & \frac{1}{2} \epsilon_{\mu \nu \rho \sigma} \partial_{\nu} n_{\rho \sigma}^{(n)}\left(s_{n}+\hat{\mu}\right) \\
= & \sum_{i, j, l=0}^{n-1} k_{\mu}\left(n s_{n}\right. \\
& +(n-1) \hat{\mu}+i \hat{\nu}+j \hat{\rho}+l \hat{\sigma}), \\
n_{\rho \sigma}^{(n)}\left(s_{n}\right)= & \sum_{i, j=0}^{n-1} n_{\rho \sigma}\left(n s_{n}+i \hat{\rho}+j \hat{\sigma}\right),
\end{aligned}
$$

where $s_{n}$ is a site number on the reduced lattice. For example,

$$
\begin{aligned}
k_{\mu}^{(2)}\left(s_{2}\right) & =\sum_{i, j, l=0}^{1} k_{\mu}\left(2 s_{2}+\hat{\mu}+i \hat{\nu}+j \hat{\rho}+l \hat{\sigma}\right), \\
k_{\mu}^{(4)}\left(s_{4}\right) & =\sum_{i, j, l=0}^{3} k_{\mu}\left(4 s_{4}+3 \hat{\mu}+i \hat{\nu}+j \hat{\rho}+l \hat{\sigma}\right) \\
& =\sum_{i, j, l=0}^{1} k_{\mu}^{(2)}\left(2 s_{4}+\hat{\mu}+i \hat{\nu}+j \hat{\rho}+l \hat{\sigma}\right) .
\end{aligned}
$$

These equations show that the relation between $k_{\mu}^{(4)}\left(s_{4}\right)$ and $k_{\mu}^{(2)}\left(s_{2}\right)$ is similar to that between $k_{\mu}^{(2)}\left(s_{2}\right)$ and $k_{\mu}(s)$ and hence one can see the above Eq. (41) corresponds to the usual block-spin transformation. After the block-spin transformation, the number of short lattice artifact loops decreases while loops having larger magnetic charges appear. We show an example of the loop length and loop number distribution of the four step $(n=4)$ blocked monopoles in Table III with respect to the same original vacuum as in Table II. For reference, we show the relation between the spacing of the blocked lattice and $\beta$ in Fig. 1.
TABLE III. The $n=4$ blocked monopole loop distribution [Loop length (L) vs Loop number (No.)] in various gauges on $6^{4}$ reduced lattice volume at $\beta=3.6$ in the same vacuum used in Table II.

\begin{tabular}{lccrcc}
\hline \hline NGF I $=1$ & & MCG I $=1$ & \multicolumn{3}{c}{ DLCG I $=1$} \\
$\mathrm{~L}$ & No & $\mathrm{L}$ & No & $\mathrm{L}$ & No \\
\hline 9266 & 1 & 4 & 5 & 4 & 8 \\
& & 6 & 1 & 6 & 2 \\
& & 10 & 1 & 406 & 1 \\
& & 340 & 1 & & \\
\hline AWL I $=1$ & & MAU1 I $=1$ & & MAU1 I $=3$ & \\
L & No & L & No & L & No \\
\hline 4 & 5 & 4 & 12 & 4 & 8 \\
6 & 1 & 6 & 1 & 6 & 3 \\
14 & 1 & 10 & 1 & 8 & 2 \\
352 & 1 & 24 & 1 & 16 & 1 \\
& & 26 & 1 & 276 & 1 \\
& & 270 & 1 & & \\
\hline \hline
\end{tabular}

In Fig. 1 and in what follows we present spacings $a$ and $b$ in units of $1 / \sqrt{\sigma}$.

\section{Numerical results}

Now let us show the simulation results with respect to VNABI (Abelian-like monopole) densities. Since monopoles are three-dimensional objects, the density is defined as follows:

$$
\rho=\frac{\sum_{\mu, s_{n}} \sqrt{\sum_{a}\left(k_{\mu}^{a}\left(s_{n}\right)\right)^{2}}}{4 \sqrt{3} V_{n} b^{3}},
$$

where $V_{n}=V / n^{4}$ is the 4 dimensional volume of the reduced lattice, $b=n a(\beta)$ is the spacing of the reduced lattice after $n$-step block-spin transformation. $s_{n}$ is the site

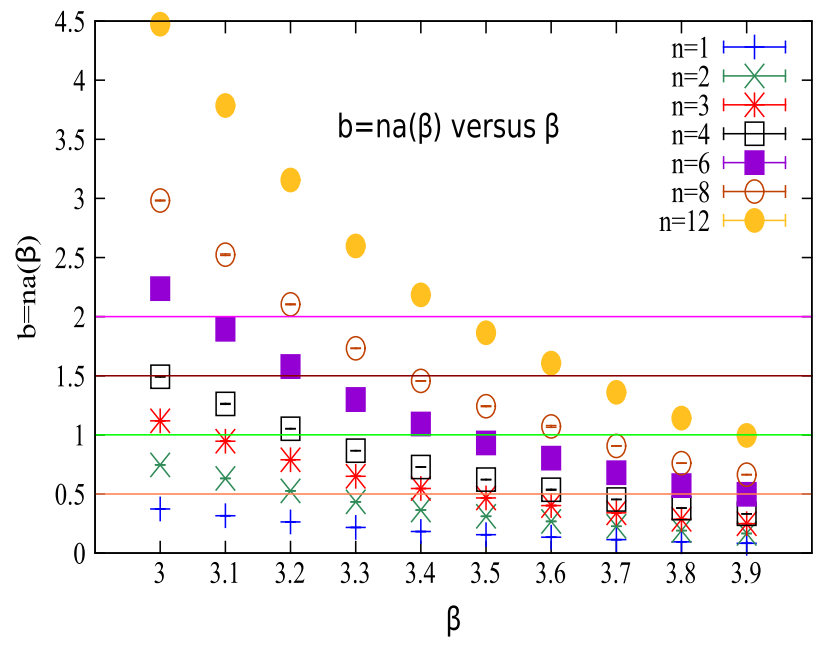

FIG. 1. $b=n a(\beta)$ in unit of $1 / \sqrt{\sigma}$ versus $\beta$. 
on the reduced lattice and the superscript $a$ denotes a color component. Note that $\sum_{a}\left(k_{\mu}^{a}\right)^{2}$ is gauge-invariant in the continuum limit. Although the global color invariance is exact except in MAU1 gauge, the average of the density of each color component of $\left|k_{\mu}^{a}\right|$ is not equal to the average of the above $\rho$, since two or three colored monopoles can run on the same dual links. In general, the density $\rho$ is a function of two variables $\beta$ and $n$.

\section{Scaling}

For the purpose of studying the continuum limit, it is usual to analyze scaling behaviors. First of all, let us show the data of MCG case in Fig. 2. In this figure and in what follows we present the monopole density $\rho$ in units of $\sigma^{1.5}$. When the scaling exists for both the string tension and the monopole density, we expect $\rho \rightarrow$ const as $a(\beta) \rightarrow 0$ and $V \rightarrow \infty$, since $a(\beta)$ is measured in unit of the string tension.
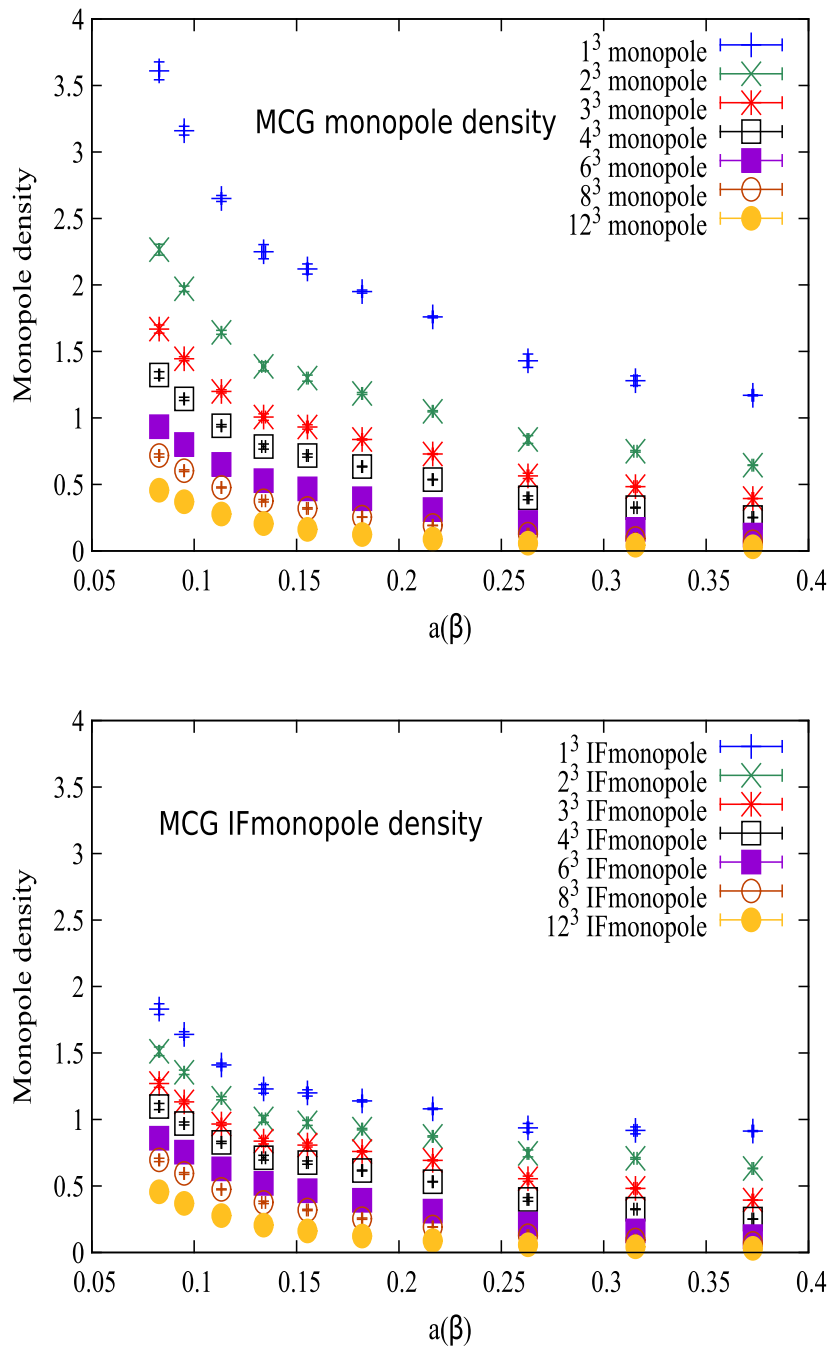

FIG. 2. The VNABI (Abelian-like monopoles) density versus $a(\beta)$ in MCG on $48^{4}$. Top: total density; bottom: infrared density. $n^{3}$ in the legend means $n$-step blocked monopoles.
In the case of total monopole density such a behavior is not seen yet. When infrared monopoles alone and blocked monopoles are considered, the behavior becomes flatter as seen from Fig. 2. But still this scaling is not conclusive. We need to study larger $\beta$ regions on larger lattice volumes. These features are very much similar in other smooth gauges as AWL, DLCG, and MAU1 and so their data are not shown here.

\section{Scaling under the block-spin transformations}

It is very interesting to see that more beautiful and clear scaling behaviors are observed when we plot $\rho(a(\beta), n)$ versus $b=n a(\beta)$. As one can see from the figures shown below for various smooth gauges considered in this work, one can see a universal function $\rho(b)$ for $\beta=3.0-3.9$ $(\beta=3.0-3.7)$ and $n=1,2,3,4,6,8,12(n=1,2,3,4,6)$ on $48^{4}\left(24^{4}\right)$ lattice. Namely $\rho(a(\beta), n)$ is a function of $b=n a(\beta)$ alone. Thus we observe clear indication of the continuum $(a(\beta) \rightarrow 0)$ limit for the lattice VNABI studied in this work.

\section{MCG case}

First we show the case of MCG gauge-fixed vacua in details. As can be seen from Fig. 9, data for $\rho(a(\beta), n)$ can be expressed by a function of one argument $b=n a(\beta)$ alone. There is a very beautiful scaling behavior for the range of $\beta=3.0-3.9$ and $n=1,2,3,4,6,8,12$. When we are restricted to long infrared monopoles alone, the density becomes substantially reduced for small $b<0.5$ region. But the scaling also can be seen except for small $b$ region as shown in Fig. 3. The violation of scaling for small $b$ region is mainly due to the ambiguity of extracting infrared monopoles. When we restrict ourselves to the data for $b \geq 0.5$, the scaling function $\rho(b)$ is obtained using the $\chi^{2}$ fit to a simple function as shown in Fig. 4:

$$
\begin{aligned}
\rho(b) & =\exp \left(a_{1}+a_{2} b+a_{3} b^{2}\right), \\
a_{1} & =0.5302(141), \quad a_{2}=-1.4756(158), \\
a_{3} & =0.1304(35) .
\end{aligned}
$$

But the fit is not good enough, since $\chi^{2} / N_{\text {dof }}=12.56$ for $N_{\text {dof }}=44$. Here we show the function (43) only for the purpose of illustration, since we have not found a simple but better fit.

To see in more details, let us consider the data points at $b=0.5,1.0,1.5,2.0$ for each $n$. Especially the data at $b=1.0$ can be fixed from the data at 5 different values of $\beta$ from $3.0 \leq \beta \leq 3.9$ as seen from Fig. 1 and Table IV. One can see the scaling behavior also clearly from the density plot for different $n$ at $b=1.0,1.5,2.0$ as shown in Fig. 5. However a scaling violation is seen at $b=0.5$ [51]. 

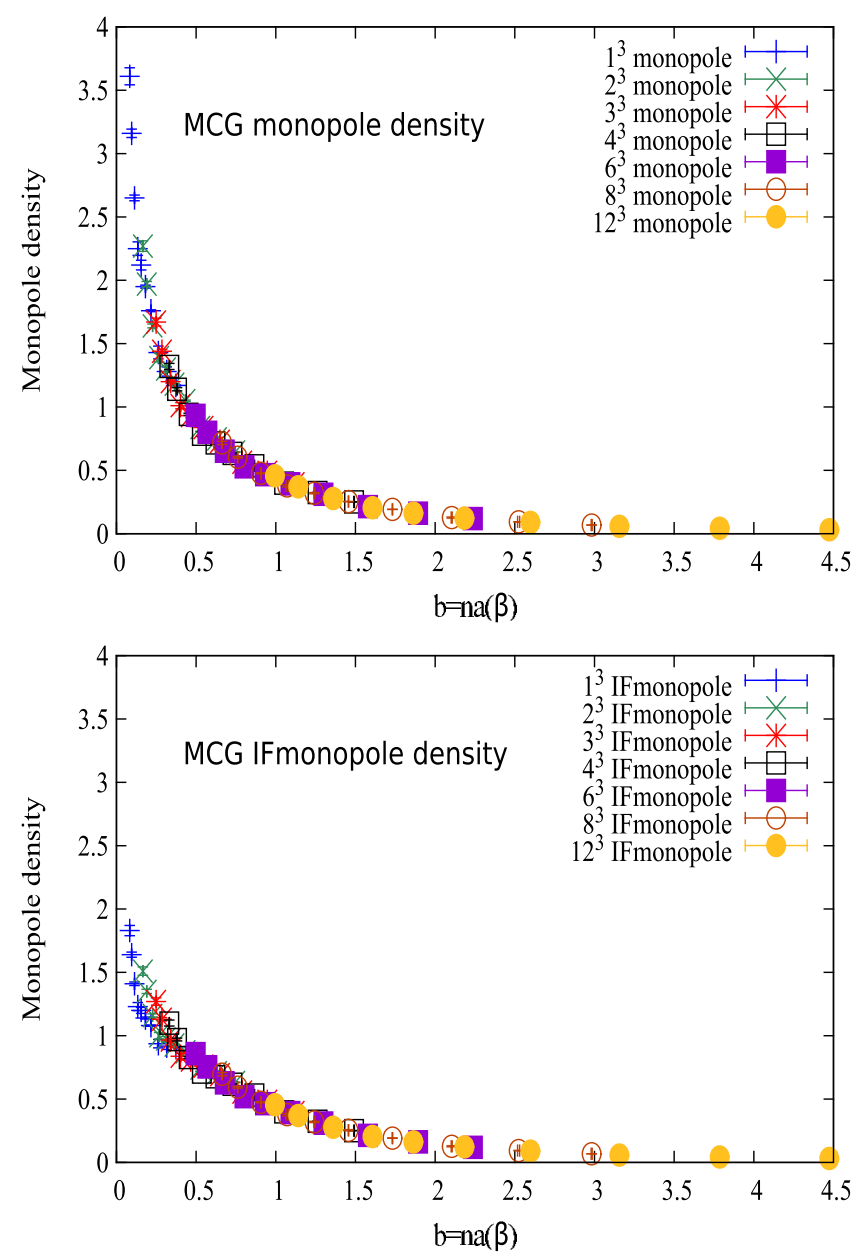

FIG. 3. The VNABI (Abelian-like monopoles) density versus $b=n a(\beta)$ in MCG on $48^{4}$. Top: total density; bottom: infrared density.

\section{AWL case}

Very similar behaviors are seen in the AWL gauge case. Again beautiful scaling behaviors for the range of $\beta=$ $3.0 \sim 3.9$ and $n=1,2,3,4,6,8,12$ are seen in Fig. 6. But in the case of infrared monopoles shown in Fig. 6, a scaling violation is observed for small $b$ region.

TABLE IV. IF monopole density $\rho_{I F}$ around $b=1.0$ for each blocking steps $n$ in MCG case on $48^{4}$.

\begin{tabular}{lrcccc}
\hline \hline$n$ & $\beta$ & $b=n a(\beta)$ & $d b$ & $\rho_{I F}$ & error \\
\hline 3 & 3.0 & 1.1184 & 0.0012 & $3.94 \mathrm{E}-01$ & $1.42 \mathrm{E}-03$ \\
3 & 3.1 & 0.9465 & 0.0024 & $4.82 \mathrm{E}-01$ & $4.06 \mathrm{E}-03$ \\
4 & 3.2 & 1.052 & 0.0016 & $3.99 \mathrm{E}-01$ & $1.40 \mathrm{E}-02$ \\
4 & 3.3 & 0.866 & 0.0008 & $5.32 \mathrm{E}-01$ & $2.37 \mathrm{E}-03$ \\
6 & 3.4 & 1.092 & 0.0012 & $3.93 \mathrm{E}-01$ & $2.80 \mathrm{E}-03$ \\
6 & 3.5 & 0.9318 & 0.0024 & $4.64 \mathrm{E}-01$ & $7.44 \mathrm{E}-03$ \\
8 & 3.6 & 1.0712 & 0.0072 & $3.77 \mathrm{E}-01$ & $9.20 \mathrm{E}-03$ \\
8 & 3.7 & 0.9064 & 0.0008 & $4.75 \mathrm{E}-01$ & $3.78 \mathrm{E}-03$ \\
12 & 3.8 & 1.1412 & 0.0012 & $3.70 \mathrm{E}-01$ & $4.43 \mathrm{E}-03$ \\
12 & 3.9 & 0.9948 & 0.0024 & $4.56 \mathrm{E}-01$ & $8.36 \mathrm{E}-03$ \\
\hline \hline
\end{tabular}

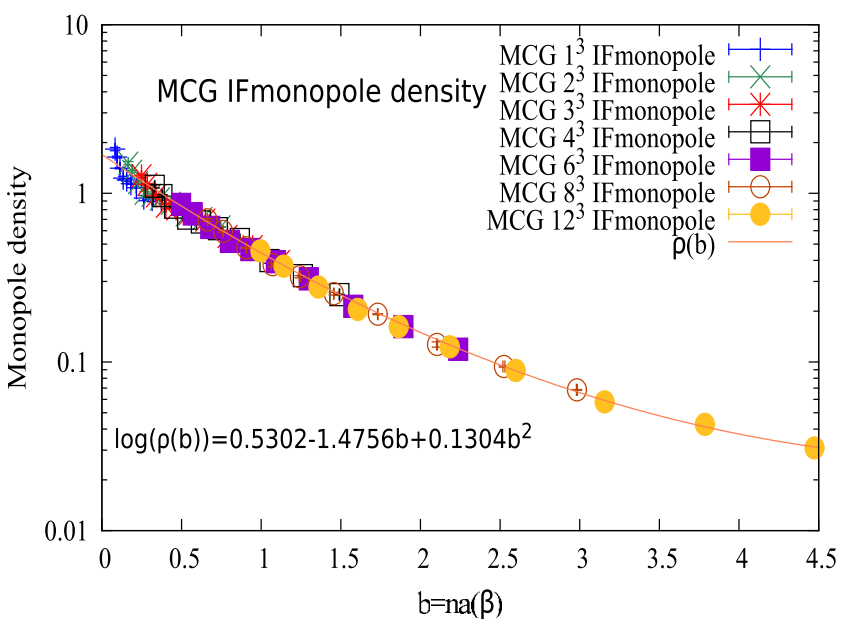

FIG. 4. The fit of the infrared VNABI (Abelian-like monopoles) density data in MCG on $48^{4}$ lattice to Eq. (43).

\section{DLCG case}

Since the DLCG gauge-fixing needs much time for larger lattice, we evaluate monopole density only on $24^{4}$ lattice. As seen from Fig. 7, a scaling behavior is found, although small deviations exist for small $b$ region.

\section{MAU1 case}

Now we discuss the case of MAU1 gauge. In this gauge, the global isospin symmetry is broken. Hence let us first evaluate the monopole density in each color direction. Namely

$$
\rho^{a}=\frac{\left.\sum_{\mu, s_{n}} \mid k_{\mu}^{a}\left(s_{n}\right)\right) \mid}{4 V_{n} b^{3}} .
$$

As expected we find $\rho^{1} \sim \rho^{2} \neq \rho^{3}$, so that we show $\rho^{2}$ and $\rho^{3}$. The results are shown in Fig. 8. Here the scaling is seen

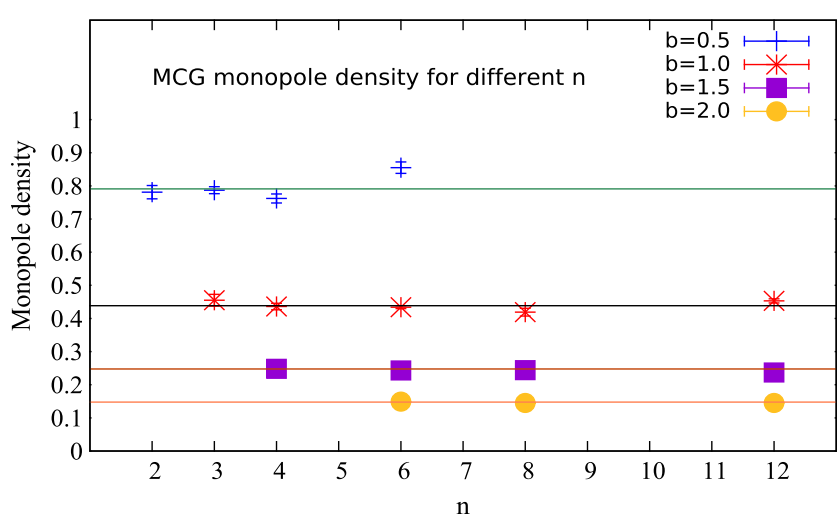

FIG. 5. The VNABI (Abelian-like monopole) density at $b=0.5,1.0,1.5,2.0$ for different $n$ in MCG on $48^{4}$. The data used are derived by a linear interpolation of two nearest data below and above for the corresponding $b$ and $n$. As an example, see the original data at $b=1.0$ in Table IV. 

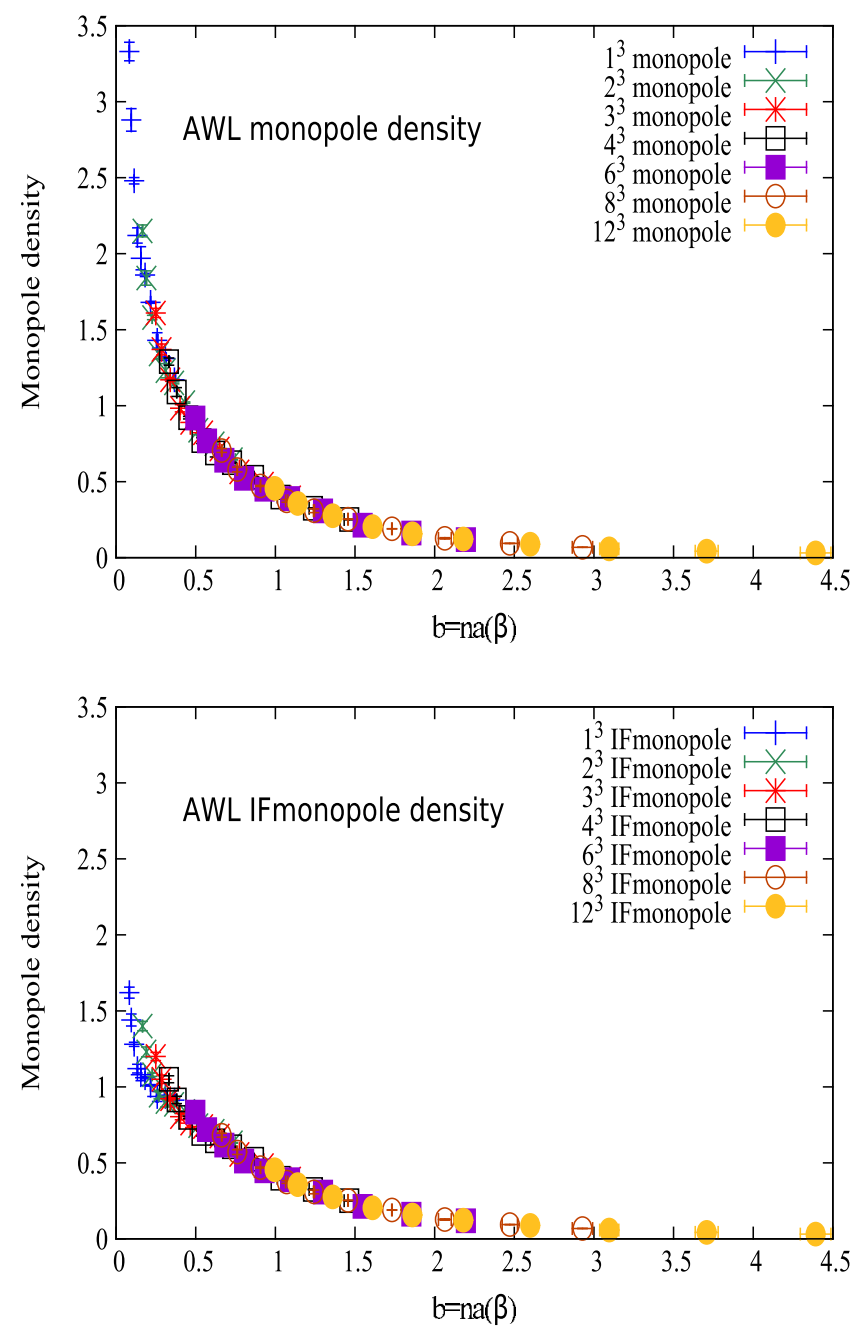

FIG. 6. The VNABI (Abelian-like monopoles) density versus $b=n a(\beta)$ in AWL on $48^{4}$. Top: total density; bottom: infrared density.

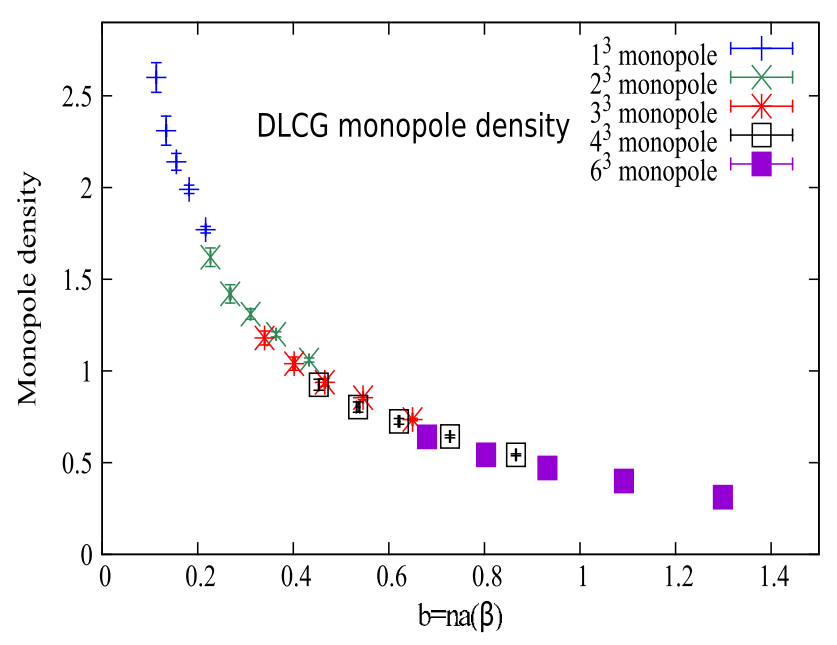

FIG. 7. The VNABI (Abelian-like monopoles) density versus $b=n a(\beta)$ in DLCG on $24^{4}$.
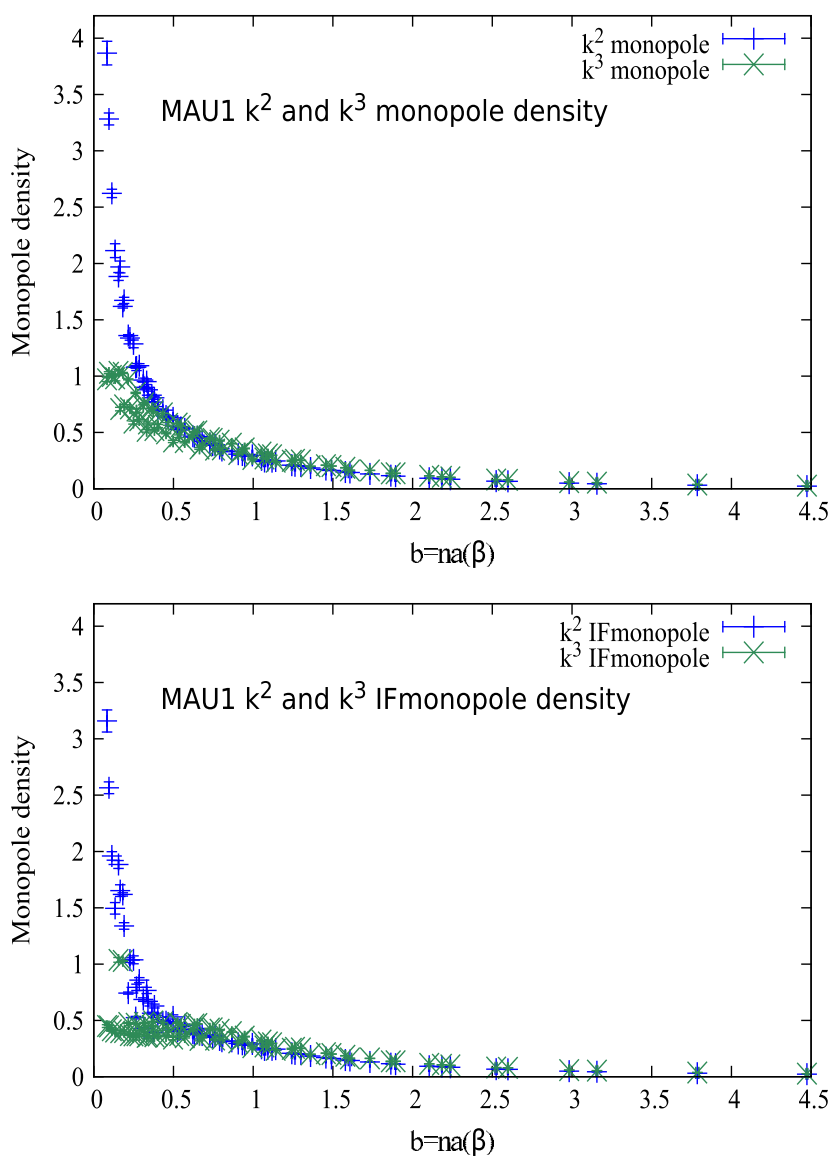

FIG. 8. The VNABI (Abelian-like monopoles) density versus $b=n a(\beta)$ for $k^{2}$ and $k^{3}$ components in MAU1 on $48^{4}$. Top: total density; bottom: infrared density.

clearly with respect to the off-diagonal $k^{2}$ currents, but the violation is seen for the diagonal $k^{3}$ currents especially at small $b$ region. Similar behaviors are found when we are restricted to infrared monopoles.

However when we evaluate the monopole density (42), we can observe similar beautiful scaling behaviors as in MCG and AWL cases. They are shown in Fig. 9.

\section{Gauge dependence}

Since $\sum_{a}\left(k_{\mu}^{a}\right)^{2}$ should be gauge-invariant according to our derivation in Sec. II, we compare the data in different smooth gauges. Look at Fig. 10, which show the comparison of the data in four gauges (MCG, AWL, DLCG and MAU1). One can see that data obtained in these four different gauges are in good agreement with each other providing strong indication of gauge independence. This is the main result of this work. Note that in MAU1 gauge, the global color invariance is broken and usually off-diagonal color components of gauge fields are said to have large lattice artifacts. However here we performed additional U1 Landau gauge-fixing with respect to the remaining $U(1)$ symmetry after MA fixing, which seems to make the vacua 

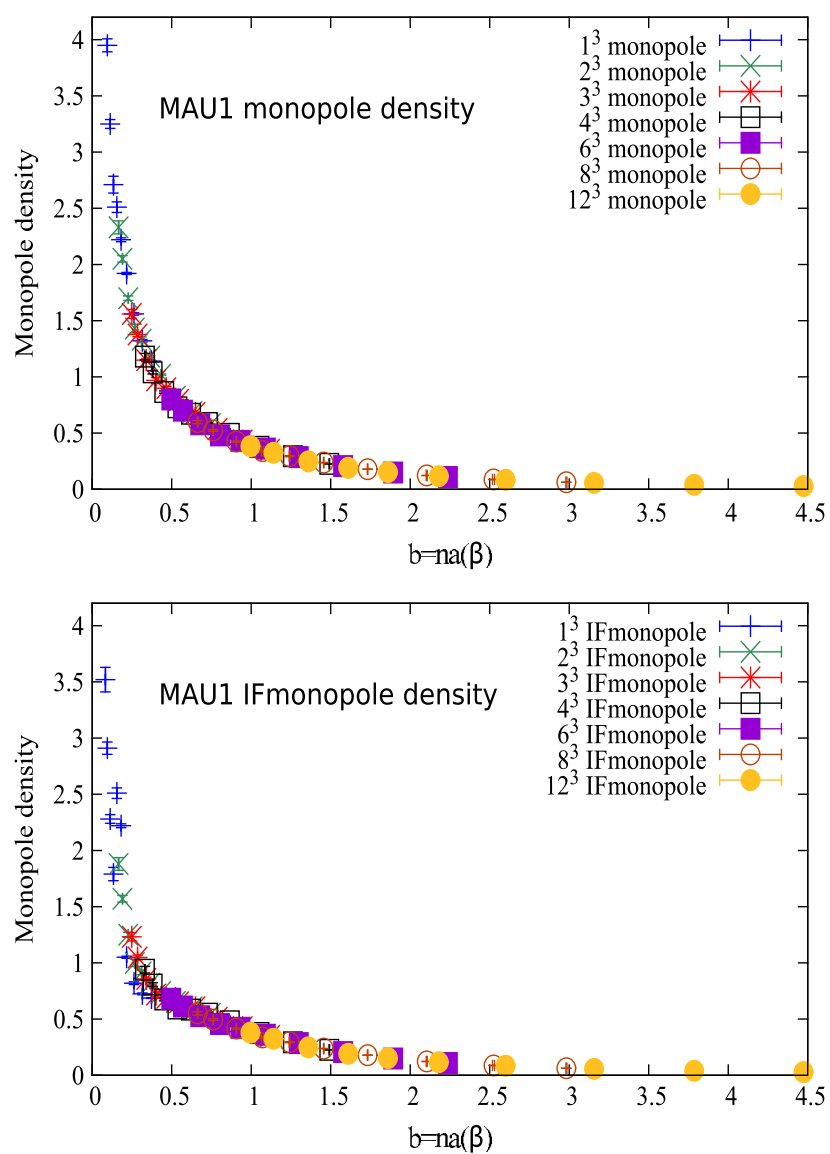

FIG. 9. The VNABI (Abelian-like monopoles) density (42) versus $b=n a(\beta)$ in MAU1 on $48^{4}$. Top: total density; bottom: infrared density.

smooth enough as those in MCG gauge case. The fact that the scaling functions $\rho(b)$ obtained in MCG gauge can reproduce other three smooth-gauge data seems to show that it is near to the smallest density corresponding to the continuum limit without large lattice artifact effects. In other non-smooth gauges such as NGF, $\rho$ does not satisfy the scaling and actually becomes much larger. This is due to our inability to suppress lattice artifacts in the nonsmooth gauges or without gauge-fixing.

\section{E. Volume dependence in MCG case}

The volume dependence is also studied when the two data on $48^{4}$ and $24^{4}$ lattices in MCG are plotted for the same $\beta$ region $(3.0 \leq \beta \leq 3.6)$ and the blocking steps $(1 \leq n \leq 6)$ as shown in Fig. 11. We found sizable finite volume effects for $\beta=3.7$ only (not shown in the figure) when lattice size for $L=24$ becomes $L a<2.7 / \sqrt{\sigma}$. Volume dependence for $(3.0 \leq \beta \leq 3.6)$ is very small as seen from Fig. 11.

\section{F. Gauge action dependence}

Let us in short check how the gauge action adopted here improves the density $\rho$ behavior by comparing the data in
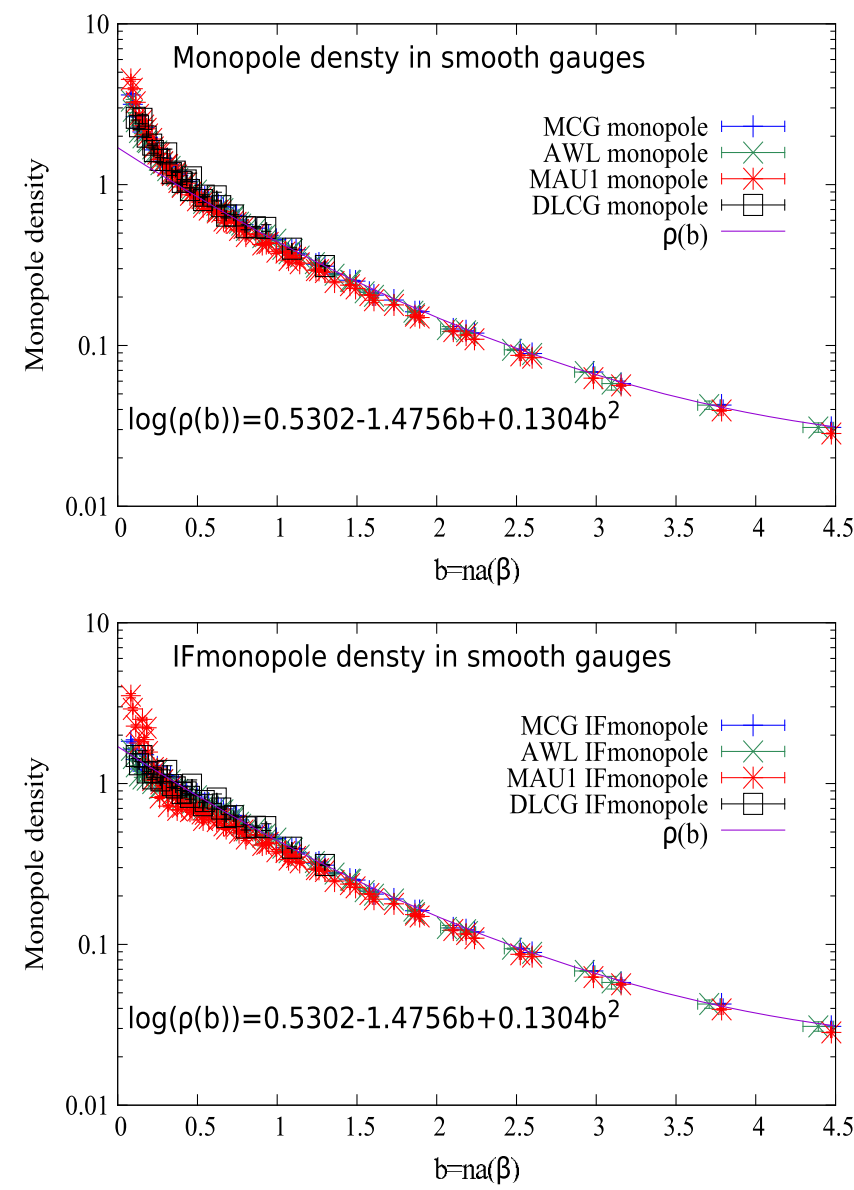

FIG. 10. Comparison of the VNABI (Abelian-like monopoles) densities versus $b=n a(\beta)$ in MCG, AWL, DLCG and MAU1 cases. DLCG data only are on $24^{4}$ lattice. Here $\rho(b)$ is a scaling function (43) determined from the Chi-Square fit to the IF monopole density data in MCG. Top: total density; bottom: infrared density.

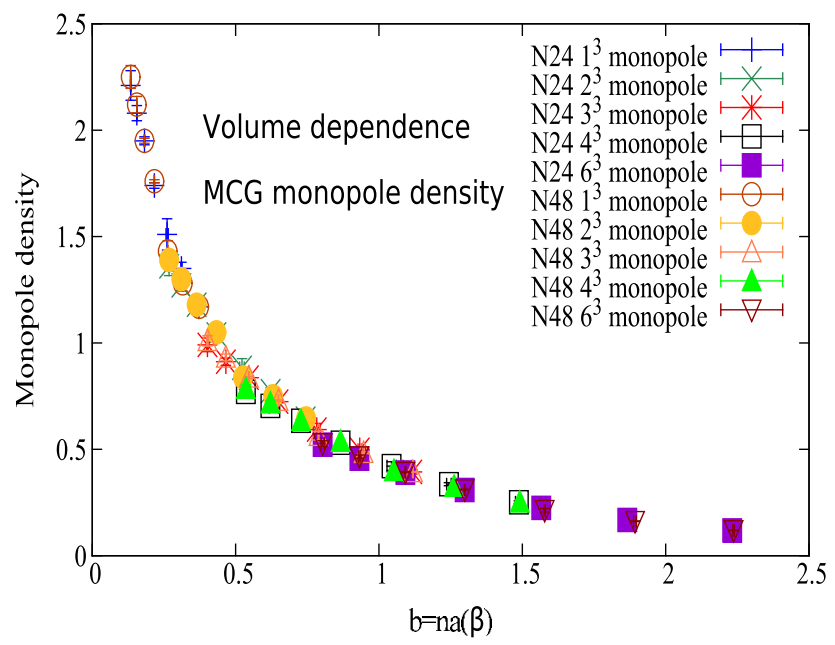

FIG. 11. Volume dependence of VNABI (Abelian-like monopole) density in the case of MCG in $48^{4}$ and $24^{4}$ tadpole improved gauge action. The data for $3.0 \leq \beta \leq 3.6$ and $1 \leq n \leq 6$ alone are plotted for comparison. 


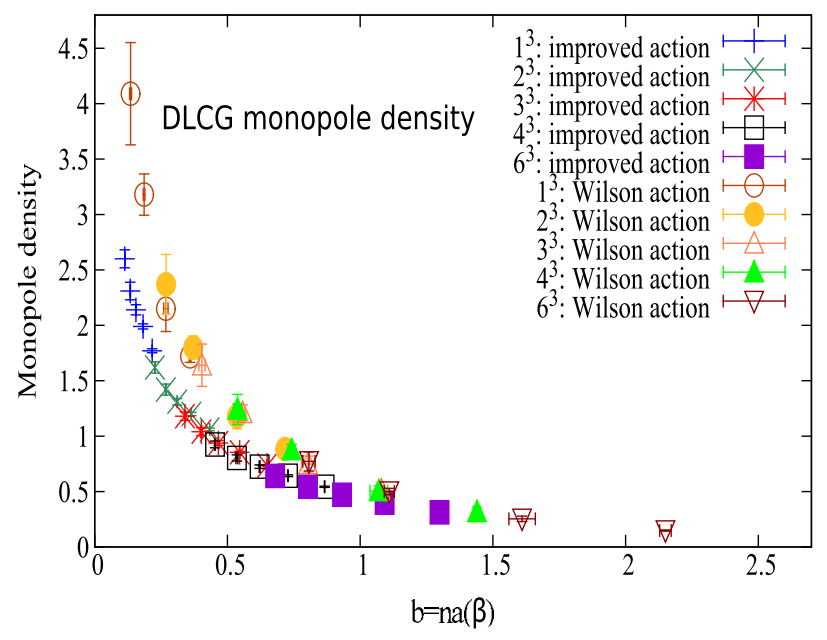

FIG. 12. Gauge action dependence of VNABI (Abelianlike monopole) densities in the case of DLCG in $24^{4}$ tadpole improved and Wilson gauge actions, The data for $3.3 \leq \beta \leq 3.7$ and $1 \leq n \leq 6$ alone are plotted.

the tadpole improved action with those in the simple Wilson gauge action. It is shown in Fig. 12. The density in the Wilson action is higher especially for $b \leq 1.0$ and so considerable improvement is obtained with the choice of the tadpole improved gauge action.

\section{CONCLUSIONS}

In conclusion, we have proposed a new color confinement scheme which is summarized as follows:

(1) VNABI is equal to the Abelian-like monopole coming from the violation of the Abelian-like Bianchi identities.

(2) VNABI satisfies the Abelian-like conservation law as well as the covariant one. Hence there are $N^{2}-1$ conserved magnetic charges in the case of color $S U(N)$.

(3) All magnetic charges are quantized à la Dirac.

(4) VNABI can be defined on lattice as lattice Abelianlike monopoles. Previous numerical results $[32,33]$ suggest that the dual Meissner effect due to condensation of VNABI must be the color confinement mechanism of QCD. The role of Abelian monopoles is played by VNABI. This must be a new scheme for color confinement in QCD.

(5) Condensation of the color invariant magnetic currents $\lambda_{\mu}$ which are the eigenvalue of VNABI $J_{\mu}$ may be a key mechanism of the physical confining vacuum.

Then to check if the new confinement scenario is correct in the continuum limit, densities of VNABI defined on lattice were studied extensively in this work. Since VNABI is equivalent to Abelian-like monopoles in the continuum, VNABI on lattice is defined as lattice Abelian-like monopoles following DeGrand-Toussaint [23]. This definition even on lattice keeps partially the topological property of VNABI satisfied in the continuum.

In the thermalized vacuum, there are plenty of lattice artifact monopoles which contribute equally to the density, so that we have adopted various improvement techniques reducing the lattice artifacts. One of them is to adopt the tadpole improved gauge action. The second is to introduce various gauges smoothing the vacuum, although gaugefixing is not necessary at all in the continuum. We have considered here four smooth gauges, MCG, DLCG, AWL, and MAU1. The third is to perform a blockspin renormalization group study.

With these improvement techniques, we have been able to get very beautiful results. First of all, in MCG, AWL and MAU1 gauges, clear scaling behaviors are observed up to the 12-step blockspin transformations for $\beta=3.0-3.9$. Namely the density $\rho(a(\beta), n)$ is a function of $b=n a(\beta)$ alone, i.e. $\rho(b)$. If such scaling behaviors are seen for $n \rightarrow \infty$, the obtained curve depending on $b=n a(\beta)$ alone corresponds to the continuum limit $a(\beta) \rightarrow 0$. It is just the renormalized trajectory. The second beautiful result is the gauge independence of the measured densities at least with respect to MCG, AWL, and MAU1 smooth gauges on $48^{4}$ and DLCG on $24^{4}$ adopted here. The gauge independence is the property expected in the continuum limit, since the observed quantity $\rho$ in (42) is gauge invariant in the continuum.

These beautiful results suggest that the lattice VNABI adopted here has the continuum limit and hence the new confinement scenario can be studied on lattice with the use of the lattice VNABI.

Let us note that monopole dominance and the dual Meissner effect due to VNABI as Abelian monopoles were shown partially without any smooth gauge fixing with the use of random gauge transformations in Refs. [32,33], although scaling behaviors were not studied enough. More extensive studies of these effects and derivation of infrared effective VNABI action using block-spin transformation in these smooth gauges discussed here and its application to analytical studies of nonperturbative quantities will appear in the near future.

\section{ACKNOWLEDGMENTS}

The numerical simulations of this work were done using computer clusters HPC and SX-ACE at Research Center for Nuclear Physics (RCNP) of Osaka University and the supercomputer at ITEP, Moscow. The authors would like to thank RCNP for their support of computer facilities. Work of V. B. was supported by Russian Foundation for Basic Research (RFBR) Grant No. 16-02-01146.

\section{APPENDIX A: TADPOLE IMPROVED ACTION}

The parameter $u_{0}$ has been iterated over a series of Monte Carlo runs in order to match the fourth root of the average plaquette $P$. The values of $u_{0}$ are shown in Table V. 
TABLE V. Details of the simulations with improved action.

\begin{tabular}{lcrlll}
\hline \hline$\beta_{\text {imp }}$ & $\mathrm{L}$ & $N_{\text {conf }}$ & \multicolumn{1}{c}{$u_{0}$} & \multicolumn{1}{c}{$\langle P\rangle^{1 / 4}$} & \multicolumn{1}{c}{$\sqrt{\sigma a^{2}}$} \\
\hline 3.0 & 24 & 100 & 0.89485 & $0.89510(3)$ & $0.372(3)$ \\
3.0 & 48 & 50 & 0.89485 & $0.89478(1)$ & $0.3728(4)$ \\
3.1 & 24 & 100 & 0.90069 & $0.90097(4)$ & $0.311(2)$ \\
3.1 & 48 & 50 & 0.90069 & $0.900688(1)$ & $0.3155(8)$ \\
3.2 & 24 & 100 & 0.90578 & $0.90601(3)$ & $0.261(4)$ \\
3.2 & 48 & 50 & 0.90578 & $0.905762(1)$ & $0.2630(4)$ \\
3.3 & 24 & 100 & 0.910151 & $0.910152(2)$ & $0.220(2)$ \\
3.3 & 48 & 50 & 0.910151 & $0.910150(1)$ & $0.2165(2)$ \\
3.4 & 24 & 100 & 0.91402 & $0.914021(1)$ & $0.1822(5)$ \\
3.4 & 48 & 50 & 0.91402 & $0.914017(1)$ & $0.1822(1)$ \\
3.5 & 24 & 100 & 0.917475 & $0.917480(1)$ & $0.1555(6)$ \\
3.5 & 48 & 50 & 0.917475 & $0.917478(1)$ & $0.1546(3)$ \\
3.6 & 24 & 100 & 0.920616 & $0.920616(1)$ & $0.1306(3)$ \\
3.6 & 48 & 50 & 0.920616 & $0.920615(1)$ & $0.1308(1)$ \\
3.7 & 24 & 100 & 0.92349 & $0.917484(2)$ & $0.1124(3)$ \\
3.7 & 48 & 50 & 0.92349 & $0.923484(1)$ & $0.1122(1)$ \\
3.8 & 48 & 50 & 0.926120 & $0.926126(1)$ & $0.0951(1)$ \\
3.9 & 48 & 50 & 0.928548 & $0.928573(1)$ & $0.0829(2)$ \\
\hline \hline
\end{tabular}

\section{APPENDIX B: THE MAXIMAL ABELIAN WILSON LOOP GAUGE}

In the maximal Abelian Wilson loop gauge (AWL),

$$
R=\sum_{s, \mu \neq \nu} \sum_{a}\left(\cos \left(\theta_{\mu \nu}^{a}(s)\right)\right.
$$

is maximized. Here $\theta_{\mu \nu}^{a}(s)$ is defined in Eq. (30).

Since the gauge transformation property of the Abelian link fields is not simple, to do the gauge-fixing efficiently is not easy. Hence we adopt a gauge fixing iteration method of a minimal gauge transformation starting from the alreadyknown smooth gauge configurations such as those in the maximal center gauge (MCG) or the direct Laplacian center gauge (DLCG) where the quantity $R$ in (B1) is known to be already large.

At the site $s$, the minimal gauge transformation is written as

$$
\begin{aligned}
U^{\prime}(s, \mu) & =e^{i \vec{\alpha}(s) \cdot \vec{\sigma}} U(s, \mu) \\
& =(1+i \vec{\alpha}(s) \cdot \vec{\sigma}) U(s, \mu)+O\left((\vec{\alpha})^{2}\right) .
\end{aligned}
$$

Hence in case of the minimal gauge transformation, we get

$$
\begin{aligned}
U_{0}^{\prime}(s, \mu) & =U_{0}(s, \mu)-\vec{\alpha}(s) \cdot \vec{U}(s, \mu) \\
\overrightarrow{U^{\prime}}(s, \mu) & =\vec{U}(s, \mu)+U_{0}(s, \mu) \vec{\alpha}(s)-\vec{\alpha} \times \vec{U}(s, \mu) .
\end{aligned}
$$

Then an Abelian link field (29) is transformed as

$$
\begin{aligned}
\theta_{\mu}^{\prime a}(s)= & \theta_{\mu}^{a}(s)+\delta_{\mu}^{a}(s), \\
\delta_{\mu}^{a}(s)= & \alpha^{a}(s) \\
& +\frac{1}{\left(U^{0}(s, \mu)\right)^{2}+\left(U^{a}(s, \mu)\right)^{2}} \\
& \times\left(U^{a}(s, \mu) \sum_{b \neq a} \alpha^{b}(s) U^{b}(s, \mu)\right. \\
& \left.-\epsilon_{a b c} U^{0}(s, \mu) U^{c}(s, \mu)\right) .
\end{aligned}
$$

The function $R$ is changed as follows:

$$
\begin{aligned}
R^{\prime}= & \sum_{a, \mu \neq \nu, s} \cos \left(\theta_{\mu \nu}^{a^{\prime}}(s)\right) \\
= & \sum_{a, \mu \neq \nu, s} \cos \left(\theta_{\mu \nu}^{a}(s)+\delta_{\mu}^{a}(s)-\delta_{\nu}^{a}(s)\right) \\
= & R-\sum_{a, \mu \neq \nu, s}\left(\delta_{\mu}^{a}(s)-\delta_{\nu}^{a}(s)\right) \sin \left(\theta_{\mu \nu}^{a}(s)\right) \\
= & R-\sum_{b} \alpha^{b}(s) A^{b}(s) \\
A^{b}(s)= & 2 \sum_{a \neq b} \sum_{\mu \neq \nu}\left(U^{b}(s, \mu)-\epsilon_{b c a} U^{c}(s, \mu)\right) \\
\quad & \times \frac{U^{0}(s, \mu) \sin \left(\theta_{\mu \nu}^{a}(s)\right)}{\left.U^{0}(s, \mu)\right)^{2}+\left(U^{a}(s, \mu)\right)^{2}} .
\end{aligned}
$$

Hence if we choose

$$
\alpha^{b}(s)=-c A^{b}(s) \quad(c>0),
$$

we get

$$
R^{\prime}=R+c \sum_{b}\left(A^{b}(s)\right)^{2} \geq R .
$$

The maximum value of $R$ is 3.0. Actually $R$ in MCG gauge for $\beta=3.3$ is around 2.508. When the parameter $c$ is taken as small as $0.005, R$ becomes $R \sim 2.512$ after four iterations and then tends to decrease. It is the vacuum adopted as the AWL vacuum. If we start from the thermalized vacuum without any smooth gauge-fixing, the large value of $R$ is not obtained with this minimal gauge transformation method. 
[1] K. Devlin, The Millennium Problems: The Seven Greatest Unsolved Mathematical Puzzles of our Time (Basic Books, New York, 2002).

[2] G. 't Hooft, in Proceedings of the EPS International, edited by A. Zichichi, , p. 1225, 1976.

[3] S. Mandelstam, II. Vortices and quark confinement in nonAbelian gauge theories, Phys. Rep. 23, 245 (1976).

[4] N. Seiberg and E. Witten, Electric-magnetic duality, monopole condensation, and confinement in $N=2$ supersymmetric Yang-Mills theory, Nucl. Phys. B426, 19 (1994).

[5] G. 't Hooft, Magnetic monopoles in unified gauge theories, Nucl. Phys. B79, 276 (1974).

[6] A. M. Polyakov, Quark confinement and topology of gauge theories, Nucl. Phys. B120, 429 (1977).

[7] G. 't Hooft, Topology of the gauge condition and new confinement phases in non-abelian gauge theories, Nucl. Phys. B190, 455 (1981).

[8] Z. F. Ezawa and A. Iwazaki, Abelian dominance and quark confinement in Yang-Mills theories, Phys. Rev. D 25, 2681 (1982).

[9] T. Suzuki, A Ginzburg-Landau type theory of quark confinement, Prog. Theor. Phys. 80, 929 (1988).

[10] S. Maedan and T. Suzuki, An infrared effective theory of quark confinement based on monopole condensation, Prog. Theor. Phys. 81, 229 (1989).

[11] T. Suzuki, Color confinement and asymptotic completeness, Prog. Theor. Phys. 69, 1827 (1983).

[12] A. S. Kronfeld, M. L. Laursen, G. Schierholz, and U. J. Wiese, Monopole condensation and color confinement, Phys. Lett. B 198, 516 (1987).

[13] A. S. Kronfeld, G. Schierholz, and U. J. Wiese, Topology and dynamics of the confinement mechanism, Nucl. Phys. B293, 461 (1987).

[14] T. Suzuki, Monopoles and confinement, Nucl. Phys. B, Proc. Suppl. 30, 176 (1993).

[15] V. Singh, D. A. Browne, and R. W. Haymaker, Structure of Abrikosov vortices in $S U(2)$ lattice gauge theory, Phys. Lett. B 306, 115 (1993).

[16] M. N. Chernodub and M. I. Polikarpov, , in Confinement, Duality and Nonperturbative Aspects of $Q C D$, edited by $\mathrm{P}$. van Baal (Plenum Press, New York, 1997), p. 387.

[17] G. S. Bali, C. Schlichter, and K. Schilling, Probing the QCD vacuum with static sources in maximal Abelian projection, Prog. Theor. Phys. Suppl. 131, 645 (1998).

[18] T. Suzuki, Low-energy effective theories from QCD, Prog. Theor. Phys. Suppl. 131, 633 (1998).

[19] Y. Koma, M. Koma, E.-M. Ilgenfritz, T. Suzuki, and M. I. Polikarpov, Duality of gauge field singularities and the structure of the flux tube in Abelian-projected $S U(2)$ gauge theory and the dual Abelian Higgs model, Phys. Rev. D 68 , 094018 (2003),

[20] Y. Koma, M. Koma, E.-M. Ilgenfritz, and T. Suzuki, Detailed study of the Abelian-projected $S U(2)$ flux tube and its dual Ginzburg-Landau analysis, Phys. Rev. D 68, 114504 (2003),

[21] T. Sekido, K. Ishiguro, Y. Koma, Y. Mori, and T. Suzuki, Abelian dominance and the dual Meissner effect in local unitary gauges in $S U(2)$ gluodynamics, Phys. Rev. D 76, 031501 (2007).
[22] T. Suzuki, A new scheme for color confinement due to violation of the non-Abelian Bianchi identities, arXiv: 1402.1294.

[23] T. A. DeGrand and D. Toussaint, Topological excitations and Monte Carlo simulation of Abelian gauge theory, Phys. Rev. D 22, 2478 (1980).

[24] L. Del Debbio, M. Faber, J. Greensite, and S. Olejnik, Center dominance and $Z_{2}$ vortices in $S U(2)$ lattice gauge theory, Phys. Rev. D 55, 2298 (1997).

[25] L. Del Debbio, M. Faber, J. Giedt, J. Greensite, and S. Olejnik, Detection of center vortices in the lattice Yang-Mills vacuum, Phys. Rev. D 58, 094501 (1998).

[26] C. Bonati, A. Di Giacomo, L. Lepori, and F. Pucci, Monopoles, Abelian projection, and gauge invariance, Phys. Rev. D 81, 085022 (2010).

[27] A. Di Giacomo and V. I. Zakharov, Surface operators and magnetic degrees of freedom in Yang-Mills theories, Phys. At. Nucl. 73, 711 (2010).

[28] J. Arafune, P. G. O. Freund, and C. J. Goebel, Topology of Higgs fields, J. Math. Phys. (N.Y.) 16, 433 (1975).

[29] S. Coleman and J. Mandula, All possible symmetries of the $S$ matrix , Phys. Rev. 159, 1251 (1967).

[30] Applying the Coleman-Mandula theorem to QCD, we note that Kugo-Ojima [31] derived a manifestly covariant and local canonical operator formalism of non-Abelian gauge theories. Although introducing an indefinite metric is inevitable, the unitarity of the physical $S$-matrix is proved. Moreover the stringlike behavior existing in gauge fields in the case of color confinement is not rejected in the framework.

[31] T. Kugo and I. Ojima, Local covariant operator formalism of non-Abelian gauge theories and quark confinement problem, Prog. Theor. Phys. Suppl. 66, 1 (1979).

[32] T. Suzuki, K. Ishiguro, Y. Koma, and T. Sekido, Gaugeindependent Abelian mechanism of color confinement in gluodynamics, Phys. Rev. D 77, 034502 (2008).

[33] T. Suzuki, M. Hasegawa, K. Ishiguro, Y. Koma, and T. Sekido, Gauge invariance of color confinement due to the dual Meissner effect caused by Abelian monopoles, Phys. Rev. D 80, 054504 (2009).

[34] P. Skala, M. Faber, and M. Zach, On the influence of colour magnetic currents on the confining properties of $S U(3)$ lattice gauge theory, Phys. Lett. B 424, 355 (1998); P. Skala, M. Faber, and M. Zach, Colour magnetic currents and the dual London equation in $S U(3)$ lattice gauge theory, Nucl. Phys. B494, 293 (1997); P. Skala, M. Faber, and M. Zach, Magnetic monopoles and dual London equation in $S U(3)$ lattice gauge theory, Nucl. Phys. B, Proc. Suppl. 49, 301 (1996).

[35] M. G. Alford, W. Dimm, G. P. Lepage, G. Hockney, and P. B. Mackenzie, Lattice QCD on small computers, Phys. Lett. B 361, 87 (1995).

[36] V. G. Bornyakov, E.-M. Ilgenfritz, and M. Muller-Preussker, Universality check of Abelian monopoles, Phys. Rev. D 72, 054511 (2005).

[37] G. I. Poulis, Scaling and confinement aspects of tadpoleimproved $S U(2)$ lattice gauge theory and its Abelian projection, Phys. Rev. D 56, 161 (1997). 
[38] A. Hasenfratz and F. Knechtli, Flavor symmetry and the static potential with hypercubic blocking, Phys. Rev. D 64, 034504 (2001).

[39] A. Hasenfratz, R. Hoffmann, and F. Knechtli, The static potential with hypercubic blocking, Nucl. Phys. B, Proc. Suppl. 106, 418 (2002).

[40] C. Gattringer, R. Hoffmann, and S. Schaefer, Setting the scale for the Luscher-Weisz action, Phys. Rev. D 65, 094503 (2002).

[41] V. G. Bornyakov et al. (DIK Collaboration), Finite temperature QCD with two flavors of nonperturbatively improved Wilson fermions, Phys. Rev. D 71, 114504 (2005).

[42] M. Albanese et al. (APE Collaboration), Glueball masses and string tension in lattice QCD, Phys. Lett. B 192, 163 (1987).

[43] The reader may wonder why a cooling or a smearing method smoothing the vacuum is not used instead of introducing a gauge-fixing. But these methods do not keep the value of the gauge action, changing the real vacuum. Hence they are not considered here.

[44] V. G. Bornyakov, D. A. Komarov, and M. I. Polikarpov, Pvortices and drama of Gribov copies, Phys. Lett. B 497, 151 (2001).
[45] M. Faber, J. Greensite, and S. Olejnik, Direct Laplacian center gauge, J. High Energy Phys. 11 (2001) 053.

[46] T. Suzuki, Y. Matsubara, S.-i. Kitahara, S. Ejiri, N. Nakamura, F. Shoji, M. Sei, S. Kato, and N. Arasaki, Three topics of monopole dynamics in Abelian projected QCD, Nucl. Phys. B, Proc. Suppl. 53, 531 (1997).

[47] G. S. Bali, V. Bornyakov, M. Muller-Preussker, and K. Schilling, Dual superconductor scenario of confinement: A systematic study of Gribov copy effects, Phys. Rev. D 54, 2863 (1996).

[48] S. Ejiri, S.-i. Kitahara, Y. Matsubara, and T. Suzuki, String tension and monopoles in $T \neq 0 S U(2)$ QCD, Phys. Lett. B 343, 304 (1995).

[49] T. L. Ivanenko, A. V. Pochinsky, and M. I. Polikarpov, Condensate of Abelian monopoles and confinement in lattice gauge theories, Phys. Lett. B 302, 458 (1993).

[50] H. Shiba and T. Suzuki, Monopole action and condensation in $S U(2)$ QCD, Phys. Lett. B 351, 519 (1995).

[51] The reduced chisquare $\chi^{2} / N_{\text {dof }}$ of the fit at $b=0.5$ is 6.26071 for $N_{\mathrm{dof}}=3$, whereas those at $b=1.0,1.5$ and 2.0 are $2.52175\left(N_{\text {dof }}=4\right), 2.80242\left(N_{\text {dof }}=3\right)$, and $2.25911\left(N_{\text {dof }}=2\right)$, respectively. 